NASA TM X: 55868

\title{
MAPPING OF THE EARTH'S \\ BOW SHOCK AND MAGNETIC TAIL- \\ BY EXPLORER 33
}

KENNETH W. BEHANNON

GPO PRICE $\$$

CFSTI PRICE(S) $\$$

Har sopy (HC)

3,00

Microfiche (MF)
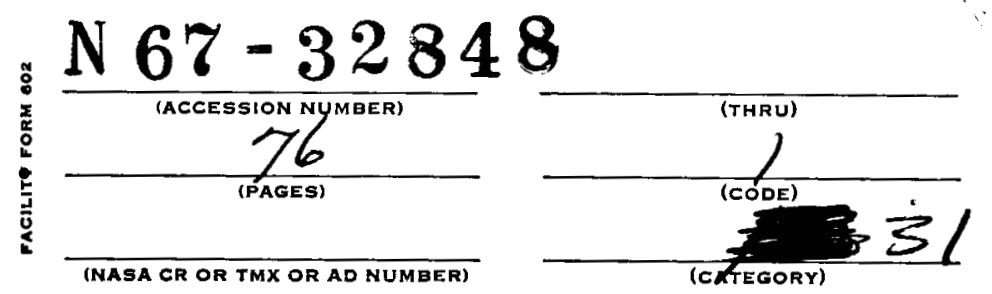

f 653 July 65

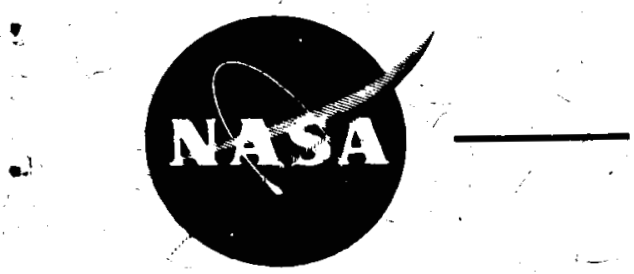

GODDARD SPACE FLIGHT CENTER

GREENBELT, MARYLAND 


\section{MAPPING OF THE EARTH'S BOW SHOCK AND} MAGNETIC TAIL BY EXPLORER 33

Kenneth W. Behannon

July 1967

\section{NASA-Goddard Space Flight Center Greenbelt, Maryland}




\section{ABSTRACT}

The Explorer 33 satellite was launched July 1, 1966 and was injected into a highly elliptical earth orbit. The Goddard Space Flight Center magnetic field experiment on board the spacecraft consists of a triaxial fluxgate sensor with a maximum dynamic range of \pm 64 gammas and a sensitivity of \pm 0.25 gammas along each axis. Because of the initial apogee-earth-sun angle of $118^{\circ}$ west of the sun, the first 8 orbits of Explorer 33 (July 1-November 11, 1966) mapped the earth's magnetosheath and magnetic tail from the western flank of the bow shock to the eastern flank. This mapping of the geomagnetic tail out to 80 earth radii established that the tail extends beyond the lunar orbital distance. Explorer 33 has also found that the earth's bow shock is still a detectable boundary between the interplanetary magnetic field and the downstream magnetosheath at a geocentric distance of 75.7 earth radii. This spacecraft has further revealed that the cross section of the geomagnetic tail is probably not cylindrical, and that the magnetic field magnitude in the tail decreases with distance down the tail from the earth. This magnitude decrease can be due both to a gradual expansion of the tail with distance and to a reconnection of magnetic field lines across the tail neutral sheet. 


\section{INTRODUCTION}

The Explorer 33 spacecraft was launched from the Eastern Test Range, Cape Kennedy, Florida on July 1, 1966 at 1602:25.375 Universal Time. The 57.1 kilogram satellite shown in Figure 1 was the first of the Anchored Interplanetary Monitoring Platform (AIMP) series and had as its primary mission to be placed into a captive lunar orbit to investigate the magnetic field, plasma and energetic particle environment of the moon.

Explorer 33 was boosted into a transfer orbit by a three-stage Thrust Augmented Improved Delta launch vehicle. Subsequent real time analysis of tracking data showed that the injection velocity following third stage burnout was $0.2 \%$ above nominal. This excess velocity was sufficient to make it impossible to achieve a stable lunar orbit since the spacecraft had no attitude control capability. The decision was made at 2100 UT to abort the primary mission and switch to the alternate mission. The alternate mission was planned in advance for the small number of possible lunar transfer trajectories in which a successful lunar orbit could not be achieved.

By firing the fourth stage retrorocket at 2233 UT, a highly elliptical earth orbit was selected which would carry the satellite to a predicted apogee of approximately $450,000 \mathrm{~km}$, well beyond the lunar orbital distance. The line of apsides of this initial orbit would have a geocentric solar ecliptic longitude $\phi_{\mathrm{SE}}$ of approximately $240^{\circ}$. The geocentric solar ecliptic coordinate system is oriented so that the $X_{S E}$ axis points from the earth to the sun, the $Z_{S E}$ axis is normal to the solar ecliptic plane, and $\mathrm{Y}_{\mathrm{SE}}$ is chosen to form a right-handed coordinate system. Geocentric solar ecliptic longitude $\phi_{\mathrm{SE}}$ is measured 
counterclockwise from the $\mathrm{X}_{\mathrm{SE}}$ axis in the ecliptic plane and $\theta_{\mathrm{SE}}$ is the corresponding latitude or angular distance above or below the ecliptic plane.

With the line of apsides initially $120^{\circ}$ west of the sun, the earth's orbital motion about the sun would sweep the orbit of Explorer 33 across the magnetic tail of the earth during the first six months after launch. The actual initial apogee was reached on July 7 at a geocentric distance of $440,300 \mathrm{~km}$ and solar ecliptic longitude and latitude of $\phi_{\mathrm{SE}}=242^{\circ}$ and $\theta_{\mathrm{SE}}=-1^{\circ}$, respectively.

The Explorer 33 spacecraft was spin stabilized at an initial rate of 26.5 rpm with a spin axis orientation of $\phi_{\mathrm{SE}}=129^{\circ}$ and $\theta_{\mathrm{SE}}=-4^{\circ}$. The initial angle between the spin axis and a line from the satellite to the sun was approximately $118^{\circ}$. The torque effects associated with solar radiation pressure on the spinning spacecraft and its solar paddles produced a subsequent precession of the spin axis in an inertial frame of reference and a continuous change in spin rate.

Figure 2 shows the variations in spin rate, spin axis-sun angle and apogeeearth-sun angle during the first five months in orbit. As can be seen the spin rate increased until the spin axis-sun angle decreased to below $90^{\circ}$ on August 10. The spin rate then decreased for the remainder of this period of solar aspect between $0^{\circ}$ and $90^{\circ}$. The orientation of the spin axis in solar ecliptic coordinates at the end of November was $\phi_{\mathrm{SE}}=339^{\circ}$ and $\theta_{\mathrm{SE}}=-5^{\circ}$. It is to be noted then that the spacecraft spin axis was nearly parallel to the plane of the ecliptic throughout the period under discussion. It will remain so during the spacecraft lifetime. 
During the first 19 weeks of the lifetime of Explorer 33, the satellite completed eight orbits about the earth, with a mean orbital period of 16.6 days. In Figure 3 is shown the projection of the initial eight orbits on the solar ecliptic plane. Also sketched in for reference are the collisionless magnetohydrodynamic bow shock wave and the magnetosphere boundary or magnetopause. The bow shock and magnetopause shown in Figure 3 are approximate boundaries for the case of cylindrical symmetry about the earth-sun line. The boundary topology actually observed by Explorer 33 is discussed in Section V. As can be seen in Figure 3, the apparent precession of the Explorer 33 orbit carried the spacecraft apogee across the magnetic tail from the western flank of the earth's bow shock to the eastern flank. The day numbers used in the figure are decimal-dayof-the-year numbers. In this system January 1 is day 0 and July 1 is day 181 . Distances are in units of earth radii $\left(1 \mathrm{R}_{\mathrm{E}}=6378.2 \mathrm{~km}\right)$.

Because of its high apogee the Explorer 33 orbit is highly sensitive to the gravitational attraction of the moon. The orbital motion of the satellite was strongly perturbed near apogee of the first orbit when the moon passed within $36,000 \mathrm{~km}$ of the spacecraft on July 8. This first lunar encounter was the closest approach of the satellite to the moon that occurred during the first ten months in orbit. The line of apsides was rotated upward across the ecliptic plane, moving the apogee of the orbit to $\theta_{\mathrm{SE}}=2^{\circ}$ for the second orbit. Figure 4 shows the projection of the initial four orbits on the noon-midnight meridian $\left(\mathrm{X}_{\mathrm{SE}}, \mathrm{Z}_{\mathrm{SE}}\right)$ plane. The effects of the lunar perturbation on Julv 8 are clearly seen.

Less drastic orbital changes occurred when Explorer 33 was within 91,500 $\mathrm{km}$ of the moon on August 2 (orbit 3 - decimal day 213) and within $60,000 \mathrm{~km}$ of 
the moon on September 27 (orbit 6 - decimal day 269). The line of apsides moved downward after orbit 2, shifting the apogee latitude $\theta_{\mathrm{A}}$ southward to a minimum of $-6^{\circ}$ in orbit 7 . On orbits 4 and 5 the apogee distance increased to $514,000 \mathrm{~km}$, or greater than $80 \mathrm{R}_{\mathrm{E}}$. During the first eight orbits the perigee distance was a minimum of $50,000 \mathrm{~km}$ on July 13 (day 193) and a maximum of $96,500 \mathrm{~km}$ on October 23 (day 295).

The Explorer 33 spacecraft was in interplanetary space 29 days out of the first 133 days in orbit. Solar-associated events monitored by the satellite during the interplanetary phases of the initial eight orbits included the passage of a shock wave on July 8 propagating outward from a solar flare (Van Allen and Ness, 1967). Because of the extensive coverage of the geomagnetosphere and magnetosheath provided by the unique Explorer 33 orbit, much of the general topology of the outer magnetosphere during the period July 1 - November 10 , 1966, may be deduced from the observations made by this satellite. It is the purpose of this paper to discuss the initial results from the magnetosphere and bow shock measurements made by the Goddard Space Flight Center (GSFC) magnetic field experiment on board Explorer 33 during that time period.

A brief review of previous pertinent investigations of the outer magnetosphere and bow shock is presented in Section II. The instrumentation of the Explorer 33 magnetic experiment is described in Section III, and the data reduction and analysis are discussed in Section IV. Initial experimental results for orbits 1-8 are presented in Section $V$. In addition to a general description of the topology of the outer magnetosphere and collisionless shock wave, detailed results from measurements in the geomagnetic tail are presented. Magnetic measurements by 
Explorer 33 have demonstrated that the earth's magnetic tail extends beyond the orbital distance of the moon (Ness et al., 1967a). Moreover, the topology of the tail at that distance is similar to that found previously at half the lunar distance by IMP 1 (Ness, 1965).

The gross geometry of the magnetic tail is described and significant spatial and temporal variations of the magnetic field in the tail are discussed. Magnetic disturbance or storm variations observed in the magnetic tail are correlated with variations seen in the horizontal component of the geomagnetic field at the surface of the earth and with the planetary magnetic activity index $K_{p}$. All of these results were described earlier in an oral report and subsequently have been discussed with much detail in a thesis (Behannon, 1967). 


\section{HISTORICAL BACKGROUND}

The first comprehensive mapping of the confined geomagnetic field, including its boundary and bow shock on the sunward side and its magnetic tail in the antisolar direction, was performed in 1963-1964 by the IMP 1 (Explorer 18) satellite (Ness et al., 1964, 1966). The corresponding energetic particle and plasma observations have been described by Bridge et al., (1965), Anderson et al., (1965), Fan et al., (1966), Wolfe et al., (1966), and Serbu (1965).

Measurements performed in the earth's magnetic tail out to $30 R_{E}$ by IMP-1 established that the tail is a permanent extension of the geomagnetosphere (Ness, 1965). An average tail field magnitude of 16 gammas was found. These measurements further provided the first experimental detection of the neutral sheet in the geomagnetic tail (Ness, 1965; Speiser and Ness, 1967). In a second inspection of earlier Explorer 14 measurements in the magnetic tail, Cahill (1966) also found examples of field reversal undoubtedly associated with the neutral sheet. A broad region of low magnetic field magnitude rather than a sharp transition was generally found. Since the Explorer 14 measurements were made nearer to the earth than the IMP 1 observations, they suggested that the field reversal region becomes thinner with increasing geocentric distance, approaching a physically real "neutral sheet" at great distances.

Measurements by Explorer 14 revealed the existence of an "electron tail" on the night side of the earth (Frank, 1965). Gringauz et al. (1960), Freeman (1964), McDiarmid and Burrows (1965), Singer et al., (1965) and Montgomery et al., (1965) all have found enhanced particle fluxes in the antisolar region of the magnetosphere, and the plasma sheet associated with the magnetically 
neutral sheet has been detected (Bame et al., 1966,1967). Isolated bursts of electrons $(E>30 \mathrm{keV}$ ) were observed in the geomagnetic tail by IMP 1 (Anderson, 1965). Sharp decreases in the magnetic field magnitude were found to accompany these electron events (Anderson and Ness, 1966).

During 1964-66 continued mapping of the magnetopause and bow shock positions was provided by the Vela 2 satellites (Gosling et al., 1967), IMP 2 (Fairfield and Ness, 1966; Serbu and Maier, 1966), and OGO 1 (Heppner et al., 1965; Heppner, 1965; Holzer et al., 1966; Heppner et al., 1967). The Vela satellites extended measurements of the boundary positions to solar ecliptic latitudes of $\pm 60^{\circ}$. Definitive mapping on the dawn side of the magnetosphere by IMP 2 demonstrated the distortion of magnetic field lines in that region and the gradual formation of the magnetic tail (Fairfield and Ness, 1967).

An east-west asymmetry of the magnetopause and the bow shock about the earth-sun line was anticipated theoretically on the basis of both the small $\left(2^{\circ}-5^{\circ}\right)$ aberration of the solar wind direction caused by the earth's motion about the sun and the effect of the oblique interplanetary magnetic field (Walters, 1964). The existence of an asymmetry of both the magnetopause and the bow shock has been experimentally verified by the Vela 2 measurements (Bame et al., 1964; Coon, 1966; Gosling et al., 1967) and by OGO 1 (Heppner, 1965; Holzer et al., 1966; Heppner et al., 1967) and IMP 3 (Ness, 1966). The center of symmetry was observed to lie approximately $2-4^{\circ}$ west of the earth-sun line.

The three-dimensional shape of the magnetopause has not yet been completely determined by space measurements. Some theories of the shape of the magnetosphere indicate that it should be slightly flattened toward the poles near the bow 
of the magnetosphere and perhaps elongated in a north-south direction further downstream (Beard, 1964). Although the Vela observations have extended measurements to much higher latitudes than reached by previous experiments, those results only have permitted a conservative limit to be set on the amount of flattening of the day-side magnetopause at high latitudes (Gosling et al., 1967).

Widely different estimates have been advanced concerning the length of the earth's magnetic tail. Dessler (1964) has calculated that the shortest possible length for the magnetospheric tail would be of the order of $100 R_{E}$. He has argued, however, that hydromagnetic wave radiation pressure will keep the tail open to a distance as great as 20-50 astronomical units. On the basis of the length of time that terrestrial lines of force being swept back into the tail will remain attached to interplanetary field lines, Dungey (1965) has estimated a much more conservative geomagnetic tail length of approximately $1000 \mathrm{R}_{\mathrm{E}^{*}}$

The first opportunity to detect the tail far downstream in the solar wind experimentally was provided by Mariner 4, which spent seven days of flight within a geocentric angle between $1^{\circ}$ and $5^{\circ}$ from the presumed center line of the tail at a radial distance of approximately $3300 R_{E}$ (Van Allen, 1965). The Geiger tube detectors on board failed to detect any effects of a magnetospheric tail during that week of favorable trajectory orientation. The Pioneer 7 spacecraft passed through the downstream region of solar wind interaction with the geomagnetic field at a geocentric distance of approximately $1000 R_{E}$ in September 1966. Detailed magnetic field measurements by Pioneer 7 (Ness et al., 1967b) suggest that magnetic lines of force connecting to the earth through the geomagnetic tail were observed. However, this preliminary inspection of the data did not reveal a coherent, well-defined tail at that distance. 
Large temporal field variations or "magnetic storms" were observed in the earth's tail by IMP 1 and were correlated with the corresponding disturbance variations recorded by magnetic observatories at the earth's surface (Behannon and Ness, 1966). A positive correlation was found between the tail field magnitude and the planetary magnetic activity index $\mathrm{Kp}$.

A correlation of the IMP 1 storm observations with measurements performed at an altitude of $1100 \mathrm{~km}$ by the APL satellite $196338 \mathrm{C}$ demonstrated the collapse of the outer trapping boundary to lower latitudes following the onset of a magnetic disturbance (Ness and Williams, 1966). These results suggest that additional lines of force are extended into the tail during a magnetic storm. Other satellite observations of the geomagnetic field during magnetic storms have been discussed by Smith and Sonett (1962), Smith et al. (1964), and Cahill and Bailey (1967). 


\section{EXPERIMENT INSTRUMENTATION}

The GSFC magnetic field experiment on board Explorer 33 utilizes a triaxial fluxgate magnetometer to provide an unambiguous measurement of the instantaneous vector magnetic field. The three sensors of the orthogonal set are mounted together as a single physical unit.

The bandpass of the electronics was chosen so that a frequency between 0 and approximately $5 \mathrm{cps}$ is passed without attenuation and all frequencies beyond are attenuated at a rate of approximately $20 \mathrm{db}$ per decade. The frequency response out to $10 \mathrm{cps}$ and the phase shift are shown in Figure 5 for one of the Explorer 33 fluxgates. The corresponding characteristics of the other two sensors are almost identical. As can be seen the response of the sensors is essentially flat over the passband containing frequencies of physical interest.

The fluxgate sensing elements can present zero scale offsets associated with sensor drift which must be determined in-flight. The unique way in which the sensors are mounted and reoriented aboard the spin-stabilized spacecraft solves this problem. In order to determine the zero level of a sensing element, it is necessary to reverse its directional sense by exactly $180^{\circ}$ while the ambient field is stationary. This experiment takes advantage of the spin stabilization of the spacecraft by mounting two of the sensors perpendicular to the spin axis as well as perpendicular to each other. Thus the basic rotation of the spacecraft reorients and thereby calibrates the zero level for two of the three axes without additional equipment.

A $90^{\circ}$ rotation of the sensor system about an axis through one of the sensors normal to the spin axis is equivalent to a $180^{\circ}$ rotation of the spin axis sensor because it interchanges the sensor initially parallel to the spin axis with one of 
the sensors initially in the equatorial plane of the satellite. Thus a thermomechanical "flipper" device which reorients the triaxial sensor set by $90^{\circ}$ provides periodic reversal of the spin axis sensor. This permits continuous monitoring and calibration of the zero level along that axis over the lifetime of the spacecraft. On Explorer 33 this reorientation is commanded once each 24 hours by the spacecraft encoder.

Stationary spacecraft magnetic fields perpendicular to the spin axis are cancelled out in the determination of the zero level shift of the two sensors which are normal to the spin axis. Thus the final accuracy of the experimental measurements is limited only by time-varying spacecraft magnetic fields for the field component perpendicular to the spin axis. However, any spacecraft field parallel to the spin axis is not cancelled out in the calibration of that sensor's zero level since the spacecraft is not inverted $180^{\circ}$ by the flipper. The only solution to this final limitation on the accuracy of the experiment is to place the sensors at a remote distance from the spacecraft on a boom support. In Figure 1 the booms and instrument canisters are shown deployed. On Explorer 33 the triaxial sensor set is 2.1 meters from the spin axis. Since this spacecraft is extremely clean magnetically already, the contamination field at the position of the sensors is reduced to the noise level of the magnetometer ( $\leq 1 / 4$ gamma) (Harris, 1966).

A block diagram of the experiment is shown in Figure 6. The three sensor data channels are sequentially gated to an analog-to-digital converter. By means of voltage comparison the 0-5 volt analog output of each of the three sensors is digitized. Following the conversion, each of the three resulting 8 bit 
words is stored in a 32 bit buffer register which also stores an 8 bit status or housekeeping word. The contents of the register are commutated to the output lines during readout period. The three axes are sampled, digitized and buffer stored in less than 15 milliseconds $(10 \mathrm{~ms}$ for the two sensors in the plane of rotation, which corresponds to $1.2^{\circ}$ of spacecraft rotation for a spin period of 3 seconds). These stored values (24 bits for all three axes) are read out four bits at a time by the telemetry data system at the scheduled times in the 81.808 second telemetry sequence.

For reasons of format simplification the magnetic field sampling was chosen to be synchronous with the telemetry rate rather than with the spin rate of the spacecraft. Because of this a measurement of the vector field is performed once every 5.11 seconds (16 measurements per telemetry sequence). This gives a sampling frequency of $0.195 \mathrm{cps}$, or for spectral analysis considerations a folding frequency of $0.0975 \mathrm{cps}$. A comparison with a typical spin modulation frequency of $0.4 \mathrm{cps}$ shows that the sampling rate is less than the spin rate, so that if there are zero level errors aliasing of the spin frequency is possible, along with any other frequency between 0.0975 and $10 \mathrm{cps}$. The effects of aliasing in the data will be discussed further in Section IV.

For this experiment the sensitivity level of the instrument was matched with the noise level of the quantization error. Extensive investigations have shown the noise level to be less than $\pm 1 / 4$ gamma. There is a maximum precision of 8 bits per axis in the analog-to-digital conversion. Since decimal numbers from 0 to 255 can be represented by 8 binary digits, the maximum possible number of magnetic field steps is 256 . With $\pm 1 / 4$ gamma per step the resulting dynamic range of the instrument is \pm 64 gammas along each axis. 
The sensitivity and linearity of each axis is checked each 12 hours by the addition of a known magnetic field parallel to the sensor axis. A special solenoidal coil carrying an accurately known current provides the field for the sensitivity checks. The performance of the magnetic field experiment for the first five months of operation was completely consistent with preflight calibrations of accuracy and precision. 
IV. DATA REDUCTION AND ANALYSIS

The GSFC magnetic field data from Explorer 33 are initially subjected to a calibration analysis. Calibrations events occur twice daily and reorientation or flip events once daily. Around these events the 16 data values in each telemetry sequence from each of the sensors in the spacecraft equatorial plane are fitted by a least squares technique, and the DC level, amplitude and phase of the fitted cosine wave as well as the root-mean-square error of the best fit are obtained (Behannon, 1967). The DC levels for each sensor are averaged to provide a daily zero level for that sensor. This information is used in the subsequent analysis of the magnetic field data. The zero levels determined in this manner are accurate to within \pm 0.2 gamma.

A check on the sensitivities of the sensors is obtained from the fit to the data around the time when the calibration currents are on at the sensors. The difference in the DC level when the current is on provides an incremental magnetic field and hence a measure of the sensitivity.

Magnetic field magnitudes are computed from each of the sets of 5.11 second measurements from the three orthogonal sensors. Sequence averages of the magnetic field magnitude and of the vector components are computed. These are linear averages over the 16 individual magnitude or component values in each 81.808 second telemetry sequence. For each average value that is computed for the total field or field components the corresponding root-mean-square deviation over the telemetry sequence period is also computed. These deviations are measures of the high frequency fluctuations of the field. The sequence average solar ecliptic field components are used to compute the 82 second average orientation 
of the magnetic field vector in terms of the azimuthal and latitudinal angles $\phi_{\mathrm{SE}}$ and $\theta_{\mathrm{SE}}$.

As stated in Section III, the quantization error of measurement of the field is \pm 0.25 gamma on each axis. This is the initial error in each component in the rotating frame of reference. An error analysis has been performed to investigate the propagation of errors through the series of coordinate transformations used in the analysis (Behannon, 1967). It is found that, on the average, the error in the field magnitude is no greater than that in each individual component $( \pm 0.25$ gamma). When the data are rotated into a fixed payload reference frame, the errors in the equatorial plane components then also include the effect of the small uncertainty in the rotation angle and in addition are directly proportional to the field strengths being measured.

For the range of magnetic field magnitudes observed by Explorer 33, from less than one gamma on each axis to nearly 64 gammas on each axis, the errors in the payload equatorial plane components range from \pm 0.25 gamma to \pm 0.40 gamma. Because it is not effected by the transformation, the error in the spin axis component remains a constant \pm 0.25 gamma.

It is further found in the error analysis that the additional transformation into solar ecliptic or solar magnetospheric coordinates does not introduce any significantly large additional errors if the spin axis orientation angles in those reference frames are accurate to within \pm 0.1 degree. In that case the errors in the solar ecliptic components are approximately equal to the errors in the payload equatorial components over the observed range of field intensities. 
Up to this point it has been assumed that no errors are introduced into the data in the conversion from engineering units to magnetic field units. For the ith component this conversion has the form

$$
\left.B_{i}(\text { gammas })=\left[B_{i} \text { (e.u. }\right)-Z_{i}\right] S_{i},
$$

where $Z_{i}$ is the zero level for the ith component in engineering units and $S_{i}$ is the slope of the linear part of the calibration curve for that sensor (which is valid over the range of field magnitudes being measured).

As can be seen an error in the slope would produce an error in the converted data which is directly proportional to the component magnitude being converted. An example of the generally good agreement between the results of the in-flight calibration checks and the laboratory and test coil facility calibration of the sensors is shown in Figure 7. The difference between the output of one of the sensors when the calibration current is on and when it is off is shown as a function of the component of the field being measured. In this case it is the component parallel to the spin axis. The component magnitudes are in units of counts (engineering units) and are sequence averages. The constant value of the difference over the range of observed field strengths is seen to justify the assumption of a linear sensitivity. 
It can be seen that the pre-flight difference falls well within the in-flight difference limits. The pre-flight slopes have been used in the conversion of all of the measurements performed during the period under discussion.

An uncertainty in the zero levels of \pm 0.2 gamma has been stated previously. The zero level and slope errors combine to produce a resultant error of conversion that ranges from \pm 0.3 gamma to \pm 2.8 gammas for fields ranging from 6 gammas to 64 gammas. The errors in the payload components and hence also in the solar ecliptic components are then determined almost entirely by the $\pm 5 \%$ conversion uncertainty and will increase as the ambient field magnitude increases. However, since Explorer 33 spends better than $90 \%$ of its time measuring magnetic fields of total magnitude $\leq 15$ gammas, the errors in the solar ecliptic and solar magnetospheric components generally will be within \pm 0.5 gamma for at least $90 \%$ of the data.

An error in zero level results in not being able to remove all of the spin modulation from the data values after the conversion. This may be seen as follows:

Let $B_{\perp}$ be the component of the ambient magnetic field in the equatorial plane of the satellite, separated from the fixed $x_{p}$ (payload coordinate) axis by an angle $\Psi$. Then if at a given time $t$ there is an angle $\omega t$ between the $(X, Y)_{p}$ axes and the rotating $(X, Y)_{A}$ axes and there are small errors $\delta y$ and $\delta y$ in the zero levels of the sensors in the plane of rotation, then the transformation to the payload reference frame has the following result: 


$$
\begin{aligned}
{\left[\begin{array}{l}
\mathrm{BX} \\
\mathrm{BY}
\end{array}\right]_{\mathrm{p}} } & =\left[\begin{array}{rr}
\cos \omega t & -\sin \omega t \\
\sin \omega t & \cos \omega t
\end{array}\right]\left[\begin{array}{l}
\mathrm{BX} \\
\mathrm{BY}
\end{array}\right]_{A} \\
& =\left[\begin{array}{rr}
\cos \omega t & -\sin \omega t \\
\sin \omega t & \cos \omega t
\end{array}\right]\left[\begin{array}{l}
\mathrm{B}_{\perp} \cos (\Psi-\omega t)+\delta x \\
\mathrm{~B}_{\perp} \sin (\Psi-\omega t)+\delta \mathrm{y}
\end{array}\right],
\end{aligned}
$$

or

$$
\begin{aligned}
& \mathrm{BX}_{\mathrm{p}}=\mathrm{B}_{\perp} \cos \Psi+\delta \mathrm{x} \cos \omega t-\delta \mathrm{y} \sin \omega t \\
& \mathrm{BY} \mathrm{p}_{\mathrm{p}}=\mathrm{B}_{\perp} \sin \Psi+\delta \mathrm{x} \sin \omega t+\delta \mathrm{y} \cos \omega t
\end{aligned}
$$

This demonstrates that time-varying components may exist in the payload and other fixed magnetic field components if either or both of $\delta x$ and $\delta y$ are nonzero. Further, this variation will be at the spin frequency of the satellite. The sampling theorem of information theory (Goldman, 1953) states that if a function $G(t)$ contains no frequenaies higher than $W$ cycles per second, then it is completely determined by giving its ordinates at a series of points spaced $1 / 2 \mathrm{w}$ seconds apart, the series extending throughout the entire time domain. Conversely any frequencies in data sampled at a given rate that are greater than half the sampling frequency will produce the stroboscopic phenomenon of aliasing. Hence any periodic variation in the field measurements beyond the cutoff or Nyquist frequency, defined by

$$
F_{c}=F_{s} / 2=1 / 2 \Delta T
$$

will be aliased down into the portion of the spectrum below the cutoff frequency. 
The zero levels can be determined to better than \pm 0.2 gamma. This is below the digitization noise level of \pm 0.25 gamma and hence in the time domain this nonrandom oscillation in the data is not detectable. If frequency spectra are computed using these data, then even with a very minute amount of energy in this oscillation it may produce a peak above the noise. For example, a spectral peak of width $(3$ spectral estimates $)(.01 \mathrm{cps})=0.03 \mathrm{cps}$ produced by a total zero level error of 0.1 gamma would reach a power level of

$$
\frac{(.1)^{2}}{.03}=.33 \frac{\text { gamma }^{2}}{\mathrm{cps}}
$$

which is well above the noise level of between $10^{-2}$ and $10^{-3}$ gamma ${ }^{2} / \mathrm{cps}$ to be expected in these spectra.

During the first 19 weeks that Explorer 33 was in orbit the spin period ranged from 2.29 seconds to 2.58 seconds. The corresponding aliased frequencies were computed from the relation

$$
\mathrm{F}_{\mathrm{k}}=2 \mathrm{kF} \mathrm{F}_{\mathrm{c}} \pm \mathrm{F}_{0},
$$

where for a cutoff frequency of $F_{c}, F_{0}$ is the alias $\left(0 \leq F_{0}<F_{c}\right)$ of all higher frequencies $F_{k}$, $k$ ranging over all positive integers. These aliased frequencies ranged from $0.0455 \mathrm{cps}$ down to zero in week 18 and back up to $0.0036 \mathrm{cps}$ five months after launch. Thus at least during the early weeks when the aliased frequency was closest to the cutoff frequency, spectra computed from the magnetic field data will provide a sensitive measure of any small errors in the sensor zero levels. 
Subsequent to this work it was determined that the inertial coordinate spi: axis orientation angles used in the data analysis for the period July-November 1966 contained errors that varied from approximately 0.1 to 9.3 degrees in right ascension and from 0.1 to 2.2 degrees in declination. Because of this there are errors of a few degrees in the computed solar ecliptic and solar magnetospheric magnetic field vector orientation angles for the latter part of the period, but none of the conclusions presented in Section $\mathrm{V}$ are effected by these small orientation errors. 


\section{RESULTS AND CONCLUSIONS}

As shown in Figure 3, during the five month period after launch, Explorer 33 repeatedly traversed the earth's bow shock wave, magnetosheath and magnetic tail out to distances beyond the lunar orbit. This spacecraft has demonstrated that the geomagnetic tail remains well defined at geocentric distances of greater than $80 R_{E}$ downstream in the solar wind (Ness et al., 1967a). In addition it has revealed that the bow shock remains a well-defined surface far downstream, at least to geocentric distances as great as $75 R_{E^{*}}$.

Identification of the bow shock in the magnetic field data is based principally on the observation of the discontinuity in field magnitude and an increase in higher frequency fluctuations. This change in magnitude generally occurs over an interval of time of between one and three telemetry sequences (on the order of 80-250 seconds) although traversals have been observed by Explorer 33 to occur in as little as 10 seconds. This traversal time reflects the combined effects of the thickness of the shock layer and the component of relative velocity (between spacecraft and shock) normal to the shock surface.

It will be shown that the shock and magnetopause boundaries move in and out due to variations in solar wind characteristics. Thus the relative velocity is determined by both the satellite velocity and the boundary velocity. The mean velocity of the boundaries in their in and out motions is greater than the normal component of spacecraft velocity, and hence multiple traversals are observed when the satellite is in the vicinity of either surface.

Multiple shock traversals along the eastern flank of the bow shock on day 307 (October 4) are shown in Figure 8. The top two panels show the solar 
ecliptic field orientation angles $\theta_{\mathrm{SE}}$ and $\phi_{\mathrm{SE}}$. In the lower two panels $\mathrm{F}$ is the 82 second sequence average field magnitude in gammas, and RMS is the rootmean-square deviation of the total field magnitude over each 82 second sequence period. At the bottom of the figure the position of the spacecraft in solar ecliptic coordinates is given in units of earth radii.

As can be seen the satellite was more than $36 R_{E}$ downstream and also was more than $15 \mathrm{R}_{\mathrm{E}}$ below the ecliptic plane. These data show the well-defined magnitude discontinuities typically observed by Explorer 33 along the flanks of the bow shock out to geocentric distances of $75 R_{E}$ (Behannon, 1967). The relatively sharp change in magnitude is always accompanied by an increase in the magnitude RMS, indicative of high frequency fluctuations near the shock.

The magnetopause was generally observed by Explorer 33 to be well-defined along the flanks of the geomagnetic tail except when the spacecraft was within the plasma sheet when near the boundary. Figure 9 shows predominantly magnetosheath data for day 291 (October 19). The spacecraft was even further downstream than in Figure 8 and was more than $20 \mathrm{R}_{\mathrm{E}}$ below the ecliptic plane. During the first half of the day it was more than $25 \mathrm{R}_{E}$ east of the noon-midnight meridian plane. The magnetosheath field is seen to be generally characterized by $\theta_{\mathrm{SE}}$ large and negative and $\phi_{\mathrm{SE}}$ roughly parallel to the interplanetary field azimuthal angle of $330^{\circ}$ (negative sector). The alternation of $\theta_{\mathrm{SE}}$ between large positive and negative values is found to be characteristic of the downstream magnetosheath field orientation. This suggests that the filamentary bundles of field lines being swept past the magnetosphere are alternately draped above and below the magnetic tail. 
Embedded in these magnetosheath data are intervals of from $1 / 2$ hour to 2 hours of geomagnetic tail data. Thus Figure 9 illustrates multiple magnetopause traversals along the earth's magnetic tail. As can be seen, the direction of the magnetic field vector changes relatively sharply at the magnetopause to the below-the-neutral sheet orientation of $\theta_{\mathrm{SE}}$ approximately $0^{\circ}$ and $\phi_{\mathrm{SE}}$ approximately $180^{\circ}$. The field magnitude is seen to be approximately 5 gammas larger inside the tail.

Altogether 195 bow shock traversals and 136 magnetopause traversals similar to those shown in Figures 8 and 9 have been found in the Explorer 33 magnetic field data between day 181 and day 342 . The positions of as many of those traversals as could be plotted are summarized in Figure 10. The traversal positions have been rotated into the ecliptic plane, and average boundary positions have been sketched in. At the bow, west of the earth-sun line, these boundaries coincide with the average IMP 1 boundary positions. Pioneer 6 and 7 traversal positions are also shown. Magnetopause traversals occurring during times when the boundary is compressed by an enhanced solar wind are marked.

It is immediately apparent from Figure 10 that a much larger asymmetry of the boundaries is suggested by these data than has been found previously by other spacecraft with much more restricted coverage (Ness, 1966). However, it must be taken into consideration that many of the eastern flank traversals were made near the time of the maximum inclination of the geomagnetic dipole axis to the noon-midnight meridian plane (autumnal equinox), as well as at increasingly greater distances below the ecliptic plane. 
Viewing the geomagnetic tail from the earth, the segments of orbits 1-8 lying downstream of the earth are shown in Figure 11, where they have been projected into a plane perpendicular to the tail axis. Also shown is a reference circle of radius $20 R_{E}$, which would be the average position of the tail magnetopause for the case of cylindrical symmetry. The shaded region is that swept out by the estimated neutral sheet in its daily and seasonal motion during the period July through November. The lower end of each of the segments of orbits 6-8 is the inbound portion, and the large southerly latitude of those passes is clearly seen. It is significant that Explorer 33 was entering the magnetic tail on September 29-30, October 19-20, and November 7-8.

In an effort to understand the traversal data, the boundary positions were replotted in solar magnetospheric coordinates since the geomagnetic tail should have the highest degree of symmetry in that frame of reference. Shown in Figure 12 , again looking down the tail, are cross-sectional views of boundary traversals for four different distance intervals down the tail. It is evident from this figure that the boundaries move in and out by as much as $10 \mathrm{R}_{\mathbf{E}}$ in response to solar wind variations. In two instances the magnetopause was compressed to within $15 R_{E}$ of the tail axis.

Ignoring those points representing large scale deformation of the tail during sudden commencement (SC) storms, the average magnetopause positions suggest a lack of cylindrical symmetry. They suggest that the tail cross-section is elongated in the direction perpendicular to the solar magnetospheric equatorial $\left(\mathrm{X}_{\mathrm{SM}} \mathrm{Y}_{\mathrm{SM}}\right)$ plane. The distance from the tail axis to the magnetopause in that perpendicular direction is greater than the corresponding distance in the 
equatorial plane by a factor of roughly 3 to 2 . The shock traversal positions also suggest a lack of circular symmetry.

The average topology of the geomagnetic tail field during orbits $1-8$ is shown in Figures 13-15. Hourly averages were linearly computed from the 82 second sequence averages of the magnetic field components. The average solar magnetospheric components were used to plot the projections of the tail field vectors on the $\mathrm{X}_{\mathrm{sm}} Z_{\mathrm{sm}}$ and $\mathrm{X}_{\mathrm{sm}} \mathrm{Y}_{\mathrm{sm}}$-planes. As many of the hourly vectors are shown as could be plotted without excessive overlapping.

The constant antisolar orientation of the tail field below the neutral sheet with the opposite orientation above the sheet is clearly seen. The neutral sheet was generally found to lie approximately $3 \mathrm{R}_{\mathrm{E}}$ above the solar magnetospheric equatorial plane. Because the base of the neutral sheet lies near the geomagnetic equatorial plane (Speiser and Ness, 1967), the sheet would be expected to lie above the SM equatorial plane at the time of year that the neutral sheet was observed by Explorer 33 (August 18-26).

During times when conditions are relatively quiet in the solar wind (as measured by $\mathrm{Kp}$ ), the geomagnetic tail field is the steadiest magnetic field observed in space by Explorer 33. This is illustrated in Figure 16. On day 293 (October 21) the magnetic activity index $\mathrm{Kp}$ ranged between 0 and 1 , with a daily sum of $3-(\operatorname{Lincoln}, 1967)$. It is to be noted that the spacecraft traversed the midnight meridian plane on day 293 but remained at distances greater than $20 R_{E}$ below the ecliptic plane throughout the day. 
The major types of variations observed in the geomagnetic tail field by Explorer 33 during five months in orbit were the following:

(1) temporal variations during magnetic disturbances;

(2) spatial variations associated with field reversal at the neutral sheet;

(3) a general decrease in the tail field magnitude with distance down the tail due to a magnitude gradient in the $-\mathrm{X}_{\mathrm{SE}}$ direction.

Each type of variation will be discussed and illustrated in turn.

Three magnetic storms occurred while Explorer 33 was in the earth's magnetic tail. These were all sudden commencement disturbances, beginning at 1500 UT on July 15, at 0003 UT on August 1, and at 1510 UT on September 14. In all cases there was a positive correlation between variations observed in the tail field magnitude and in the horizontal component of the field at the earth's surface during the compressional phase of the storms. A positive correlation was also found between the tail field magnitude and the $\mathrm{K}_{\mathrm{p}}$ index in each case. These results are in agreement with the IMP 1 magnetic storm observations (Behannon and Ness, 1966).

The geomagnetic storm of September 14, as observed at the earth's surface, is shown in Figure 17. The horizontal component magnetogram traces from seven magnetic observatories are shown. The sudden commencement (SC) time of the disturbance onset at the surface was 1510 UT. This storm is of particular importance because of the unique position of Explorer 33 at the time of the storm. Figure 18 shows the projection of orbit 5 on both the noon-midnight meridian plane and the solar ecliptic plane. It can be seen that on September 14 the spacecraft traversed the midnight meridian plane at a geocentric distance 
down the magnetic tail of approximately $75 \mathrm{R}_{\mathrm{E}}$ and a distance below the ecliptic plane of approximately $14 \mathrm{R}_{\mathrm{E}}$. Thus the satellite was in the magnetic tail, well beyond the lunar orbital distance of approximately $60 R_{E}$, at the onset of this storm.

The satellite magnetic field data for September 14 (day 256) are shown in Figure 19. The sudden commencement occurred at $1522 \pm 2$ UT at the spacecraft, giving a delay time of $12 \pm 2$ minutes. The satellite coordinates were $\mathrm{X}_{\mathrm{SE}}=$ -73.8, $\mathrm{Y}_{\mathrm{SE}}=-0.8$ and $\mathrm{Z}_{\mathrm{SE}}=-14.6$. Thus the impulse propagation velocity parallel to the earth-sun line must have been $654 \pm 109 \mathrm{~km} / \mathrm{sec}$. An increase of 10 gammas in the tail field magnitude occurred at the commencement of this storm. Note the magnitude fluctuations with a period of approximately 20 minutes following the $\mathrm{SC}$ at the satellite.

Large field variations were also observed by Explorer 33 prior to the storm sudden commencement. As can be seen in Figure 19, the magnetic tail field was relatively steady and characteristically oriented parallel to the earthsun line in the antisolar direction until 1009 UT. At that time a disturbance began which was characterized magnetically by angular excursions and steep magnitude decreases of between 4 and 8 gammas. The solar ecliptic coordinates of the spacecraft at that time were $\mathrm{X}_{\mathrm{SE}}=-74.7, \mathrm{Y}_{\mathrm{SE}}=0.0$ and $Z_{\mathrm{SE}}=-14.2$. In addition the solar magnetospheric coordinates were $Y_{S M}=-8.1$ and $Z_{S M}=-11.7$. Thus Explorer 33 was in the midnight meridian plane but well below the solar magnetospheric equatorial plane, precluding any possible neutral sheet effects. However, the time at which the magnetic field variations began corresponds to the onset time of the detection of electrons by the MIT plasma detector (Bridge and Binsack, personal communication). 
The final magnetic field variation of interest on day 256, as shown in Figure 19, occurred approximately two hours following the sudden commencement. At that time a sharp change in the direction of the field was observed. This event is interpreted as being a sudden traversal of the magnetopause. At that time the spacecraft had reached a point approximately $15 \mathrm{R}_{\mathrm{E}}$ from the axis of the magnetic tail. Thus the enhanced solar wind had compressed the tail boundary inward to that radius. The interpretation of the observed field variation as a magnetopause traversal is supported by the detection of magnetosheath plasma by the MIT detector (Bridge and Binsack, personal communication) following the abrupt change in field direction. Also the magnitude RMS is seen to increase at that time.

The magnetopause was crossed a total of eight times during a period of approximately 12 hours before the field relaxed back to its undisturbed topology. The magnetic field data for day 257 are shown in Figure 20. As can be seen, three shorter intervals of magnetosheath field orientation and magnitude are observed during the first six hours.

The next class of geomagnetic tail field variation to be discussed is the spatial variation associated with field reversal at the neutral sheet. The neutral sheet has been defined as a region of abrupt directional change in the earth's magnetic tail field, where the magnitude decreases to a small value (Ness, 1965). It is not possible to uniquely determine the thickness of the neutral sheet for a particular traversal unless the relative velocity normal to the sheet between the spacecraft and the sheet is known. A lower limit of approximately $600 \mathrm{~km}$ was determined for the thickness from several traversals of the sheet by IMP 1 
by neglecting the velocity of the sheet (Ness, 1965). The observed diurnal wobble of the sheet about its axis due to the daily $11.7^{\circ}$ nutation of the earth's magnetic axis and the observations of multiple sheet traversals due to "flapping" of the sheet both establish that the sheet is always in motion. The results of an analysis of 42 IMP 1 neutral sheet traversals suggest that the sheet is probably less than $1 R_{E}$ thick, though evidence for an increase in thickness toward the dawn magnetopause was found (Speiser and Ness, 1967). Thus the neutral sheet is a relatively thin region of field reversal embedded in a plasma sheet that is often 4-6 times as thick (Bame et al., 1967).

Figure 11 shows that the only times that Explorer 33 was in close proximity to the neutral sheet region were when outbound on orbit 1, during the orbit 4 pass through the tail, and during the first day of the orbit 5 pass through the tail. A complete traversal of the neutral sheet by the spacecraft on orbit 4 beyond the lunar orbital distance has already been discussed in some detail (Ness et al., 1967a).

Because of the motion of the sheet, other complete traversals for short periods of time were observed during the orbit 4 pass through the tail. One such sheet crossing by Explorer 33 is shown in detail in Figure 21. Shown in the top three panels are the sequence average field orientation and magnitude for approximately one hour. The lower panel shows the variation observed in the 5.11 second magnitude measurements during the traversal. This is an example of what might be called a "sharp" traversal, in which the magnitude drops sharply to near zero accompanied by a sharp change in the azimuthal angle $\phi_{\mathrm{SE}} \cdot$ The corresponding sequence average solar magnetosphere component data are shown in Figure 22. 
Figure 23 illustrates a second type of neutral sheet traversal that was observed. In this case the field magnitude decrease is broad and shallow, with the 5.11 second magnitude data never falling below approximately 3 gammas. The fluctuating nature of the reversal of $\phi_{\mathrm{SE}}$ suggests that the motion of the sheet past the spacecraft was not steady. The corresponding solar magnetospheric component variations are shown in Figure 24. These show that when $\mathrm{BX}_{\mathrm{SM}}$ was zero, $\mathrm{BY}_{\mathrm{SM}}$ was also approximately zero but $\mathrm{BZ}_{\mathrm{SM}}$ had a positive value of approximately 1 to 2 gammas. In the previous example all components were near zero simultaneously.

These results are consistent with the view that the neutral sheet was thicker in the second case, with a northward perpendicular component of several gammas observed throughout the reversal of the field vector. Thus the neutral sheet may undergo local variations in thickness that could be either spatial or temporal.

The simplest model of the neutral sheet that can be used for computational purposes assumes that the neutral sheet is two dimensional and stationary in time except for possible spatial motion. In terms of this model it can be argued that the resulting simplification of the expressions for the time rate of change of the field components, together with Ampere's law, lead to expressions for the components of the linear current density in the sheet that involve only the total changes in the two components of the field that are parallel to the sheet (Speiser and Ness, 1967).

Initial inspection of the Explorer 33 data revealed six really complete traversals that were suitable for computation of equivalent current density 
Table 1

NEUTRAL SHEET EQUIVALENT CURRENT DENSITIES

\begin{tabular}{|c|c|c|c|c|c|c|c|c|}
\hline DAY & TIME & $\mathrm{X}_{\mathrm{SM}}$ & $\mathrm{Y}_{\mathrm{SM}}$ & $\mathrm{Z}_{\mathrm{SM}}$ & $\triangle_{\mathrm{B}}$ & $\triangle \mathrm{B}_{\mathrm{Y}}$ & $\mathrm{J}_{\mathrm{X}}\left(\mathrm{X} 10^{-2} \mathrm{amps} / \mathrm{m}\right)$ & $\mathrm{J}_{\mathrm{y}}\left(\mathrm{X} 10^{-2} \mathrm{amps} / \mathrm{m}\right)$ \\
\hline 229 & 0945 & -50.6 & 17.0 & 3.1 & 19.6 & 3.6 & 0.3 & 1.6 \\
& 1003 & -50.7 & 16.9 & 3.0 & -23.2 & 1.2 & -0.1 & 1.8 \\
& 1218 & -51.8 & 16.5 & 3.2 & 28.2 & 2.8 & 0.2 & 2.2 \\
234 & 0847 & -79.4 & -7.5 & 1.9 & 32.0 & 1.2 & 0.1 & 2.5 \\
& 1817 & -79.8 & -9.6 & -0.9 & 26.0 & 7.5 & -0.6 & 2.1 \\
235 & 1129 & -79.7 & -12.8 & 1.6 & 26.0 & 0.0 & 0.0 & 2.1 \\
\hline
\end{tabular}


from the magnetic field component variations. The resulting linear current densities are tabulated in Table 1. Also listed are the changes in the field components that were used, the distance $X_{S M}=X_{S E}$ of the spacecraft down the tail, the distance $Y_{S M}$ of the spacecraft from the tail axis at the time of the traversal, and the distance $Z_{\mathrm{SM}}$ from the solar magnetospheric equatorial plane.

As stated by Speiser and Ness (1967), errors of $\pm 10-20 \%$ in the determination of $\vec{J}$ result from the uncertainty involved in the straight line fitting of the component data to obtain the changes across the sheet. Also of course the validity of using the two dimensional model in a given case is limited by the magnitude of the observed perpendicular field component in the sheet.

The third major type of magnetic field variation observed by Explorer 33 in the geomagnetic tail is a general decrease in tail field magnitude with increasing geocentric distance down the tail. This magnitude decrease was evident in the summary plot of hourly average vectors in Figures 13-15. Figure 25 shows 256 hourly average field magnitudes as a function of distance along the earth-sun line down the tail. Only data corresponding to $\mathrm{Kp} \leq 2$ (i.e., quiet data) and data for which the spacecraft was $\geq 2 \mathrm{R}_{\mathrm{E}}$ away from the neutral sheet have been plotted.

Data from 740 hours during which Explorer 33 was clearly in the tail were subjected to a multiple correlation and regression analysis. The formulation used in this analysis has been described by Efroymson (1965). In this particular application the hourly average field magnitude was the dependent variable, while three independent variables were used. These were the earth-sun line distance 
$\mathrm{X}_{\mathrm{SE}}$ of the spacecraft down the tail, the magnetic activity index $\mathrm{K}_{\mathrm{p}}$, and the perpendicular distance $Z_{S M}$ of the satellite from the solar magnetospheric equatorial plane. The formulation is such that functions of the independent variables can also be correlated with the dependent variable. Various powers of the $\mathrm{X}_{\mathrm{SE}}$ distance were tested, and the best correlation was obtained using $\left|\mathrm{X}_{\mathrm{SE}}\right|^{-0.3 \pm 0.2}$.

The results of the analysis are tabulated in Table 2. As can be seen, the best correlation among the three independent variables used was between field magnitude and the inverse power of distance down the tail. The lack of a better correlation between magnitude and planetary index $\mathrm{Kp}$ is at least in part due to the lack of better resolution in $\mathrm{Kp}$. The data points that are circled in Figure 25 are examples of a large increase in field magnitude preceding a corresponding increase in $\mathrm{Kp}$ by one hour.

The lack of a good correlation between field magnitude and $Z_{S M}$ was expected since the tail field magnitude is observed to be reduced by the neutral sheet only at points close to or within the sheet. In addition there is generally a nonzero difference between $Z_{S M}$ and the actual perpendicular distance to the neutral sheet.

The regression coefficients obtained in the case of the "best" correlation provided a fit to 740 hourly average field magnitudes with a standard error of \pm 3.06 gammas. Limiting case best fits to the 256 data points shown in Figure 25 were obtained from the regression equations for $\left|\mathrm{X}_{\mathrm{SE}}\right|^{-0.1}$ and $\left|\mathrm{X}_{\mathrm{SE}}\right|^{-0.5}$ and are represented by the dashed curves in Figure 25. The upper curve at $X_{S E}=-10 R_{E}$ illustrates the variation of $F$ with $\left|X_{S E}\right|^{-0.5}$. The field magnitudes 
Table 2

MULTIPLE CORRELATION AND REGRESSION COEFFICIENTS FOR MAGNETIC TAIL FIELD MAGNITUDE VARIATIONS

DEPENDENT VARIABLE = Magnetic Field Magnitude F

\begin{tabular}{|c|c|c|}
\hline $\begin{array}{c}\text { INDEPENDENT } \\
\text { VARIABLE } \\
\end{array}$ & $\begin{array}{l}\text { PARTIAL CORRELATION } \\
\text { COEFFICIENT } \\
\end{array}$ & $\begin{array}{l}\text { REGRESSION } \\
\text { COEFFICIENT } \\
\end{array}$ \\
\hline$\left|\mathrm{X}_{\mathrm{SE}}\right|^{-0.3}$ & $\mathbf{r}_{\mathbf{F X} \cdot \mathbf{K Z}}=0.66$ & $69.13 \pm 2.88$ \\
\hline kp & $\mathbf{r}_{\mathrm{FK}} \cdot \mathrm{XZ}=0.46$ & $1.30 \pm 0.09$ \\
\hline \multirow[t]{3}{*}{$Z_{\text {SM }}$} & $\mathrm{r}_{\mathrm{FZ} \cdot \mathrm{XK}}=0.14$ & $0.07 \pm 0.01$ \\
\hline & $\begin{array}{l}\text { MULTIPLE CORRELATION } \\
\text { COEFFICIENT }\end{array}$ & \\
\hline & $r_{E \times A K Z}=0.69$ & \\
\hline
\end{tabular}


predicted by the regression curves in the interval between $-10 R_{E}$ and $-30 R_{E}$ are consistent with the median tail field magnitude of 16 gammas found by IMP 1 in that region (Behannon and Ness, 1966). From the 740 hours of Explorer 33 observations in the tail out to $80 R_{E}$, a median magnitude of 10 gammas was found.

The observed field magnitude gradient in the tail can result either from an increase in the cross sectional area of the tail with distance down the tail or from reconnection of field lines across the neutral sheet or possibly from a combination of the two effects (Behannon, 1967). If the cross sectional area of the tail remains constant, then the total number of field lines in the tail must diminish with distance to produce a magnitude gradient. This can occur if there is reconnection of field lines across the neutral sheet. A simple consideration of conservation of flux through cross sections at $X_{S E}=-10 R_{E}$ and at $X_{S E}=-80$ $R_{E}$ and through the neutral sheet shows that an average 2 gamma field component perpendicular to the neutral sheet would account for the total decrease in field magnitude that occurs in that distance.

However, in the interval between $-10 R_{E}$ and $-30 R_{E}$ where the greatest part of the magnitude decrease occurs, an average 7 gamma component perpendicular to the neutral sheet would be required and nothing that large is seen on the average in that region. During 230 hours of orbit 4 an average northward component of 1 gamma was observed in the tail by Explorer 33. However, it was northward in the sense of being in the direction of the positive $Z_{S M}$ axis, and the real neutral sheet may not be coplanar with the solar magnetospheric equatorial plane. Thus it appears that both a small amount of increase in the tail cross section (as yet undetected) and a small amount of reconnection at the neutral sheet may combine to produce the observed magnitude gradient. 
If one extends the computed regression curves shown in Figure 25 beyond $80 R_{E}$ it is found that the tail field magnitude will have decreased to a value of 4 gammas at a geocentric distance between 125 and $175 R_{E}$ during times of low Kp. Although the magnetic field lines in the tail are probably stretched out much further than that (Ness et al., 1967b), the tail may be less well defined beyond some distance $\leq 200 R_{E}$ if the field magnitude decreases to the quiet time interplanetary level at that distance. 


\section{SUMMARY OF RESULTS}

During the first five months in orbit, the Explorer 33 satellite made detailed measurements at the earth's bow shock, in the magnetosheath and in the geomagnetic tail. This extensive survey has confirmed and substantially extended the results of previous investigations of those regions.

Explorer 33 has found that the earth's bow shock is a well-defined surface of discontinuity at geocentric distances as great as $75 \mathrm{R}_{\mathrm{E}}$ down the flanks. The magnetosheath fields along the flanks are generally characterized by a large component perpendicular to the ecliptic plane. This component is observed to be alternately positive and negative, suggesting that filaments are observed which are alternately draped above and below the magnetospheric tail.

Explorer 33 has generally observed a large discontinuity in magnetic field direction across the tail magnetopause. In addition, traversals of that boundary have shown that the geomagnetic tail is probably not cylindrical in shape. The cross section of the tail appears to be elongated in the direction perpendicular to the neutral sheet. The average bow shock also may not be circular in cross section. The shock and magnetopause boundaries move in and out by $10 R_{E}$ or more in response to solar wind variations. The tail magnetopause is compressed to within $15 R_{E}$ of the tail axis during magnetic storms.

Explorer 33 has shown that the geomagnetic tail is still well defined beyond the lunar orbital distance. During times of low $\mathrm{Kp}$ the magnetic field in the tail is the steadiest field observed in space by Explorer 33. At various distances down the tail out to $80 R_{E}$ the neutral sheet has been observed to be a relatively 
thin region which may undergo variations in thickness which are either spatial, temporal or both. Sudden commencement magnetic storm variations observed in the magnetic tail beyond the lunar orbital distance are similar in character to the storm variations observed in the tail nearer the earth.

A general decrease in tail field magnitude with distance down the tail has been found. The observed magnitude gradient can be described by an inverse power of the geocentric distance along the earth-sun line. This magnitude decrease can be produced either by a gradual increase in the cross section of the tail with distance or by a reconnection of field lines across the neutral sheet. It may result from a combination of both mechanisms since there is no experimental evidence to support the view that either effect is sufficiently large to be solely responsible. If the tail field magnitude continues to decrease at the observed rate beyond a geocentric distance of $80 R_{E}$, then the geomagnetic tail may be less well defined beyond a distance from the earth of several hundred earth radii.

\section{ACKNOWLEDGEMENTS}

The author wishes to express his appreciation to Dr. Norman F. Ness of the Goddard Space Flight Center for his patient guidance and support throughout this work. He would also like to thank Mr. Herbert E. Haney for his substantial programming and data processing assistance and Dr. Donald H. Fairfield, Dr. Harold E. Taylor, Mr. Clell S. Scearce and Mr. William H. Mish for their contributions in the form of many helpful discussions. In addition he would like to thank Dr. H. S. Bridge and Dr. J. H. Binsack of the Massachusetts Institute of Technology for a very profitable discussion of joint results. 


\section{REFERENCES}

Anderson, K. A., Energetic electron fluxes in the tail of the geomagnetic field, J. Geophys. Res., 70, 4741-4763, 1965.

Anderson, K. A., H. K. Harris, and R. J. Paoli, Energetic electron fluxes in and beyond the earth's outer magnetosphere, J. Geophys. Res., 70, 1039$1050,1965$.

Anderson, K. A. and N. F. Ness, Correlations of magnetic fields and energetic electrons on the IMP 1 satellite, J. Geophys. Res., 71, 3705-3727, 1966.

Bame, S. J., J. R. Asbridge, H. E. Felthauser, R. A. Olson, and I. B. Strong, Electron angular, spatial, and energy distributions, measured near $17 \mathbf{R}_{\mathbf{E}}$ with an electrostatic analyzer, Trans. Am. Geophys. Union, 45, 624, 1964.

Bames, S. J., J. R. Asbridge, H. E. Felthauser, R. A. Olson, and I. B. Strong, Electrons in the plasma sheet of the earth's magnetic tail, Phys. Rev. Letters, 16, 138-142, 1966.

Bame, S. J., J. R. Asbridge, H. E. Felthauser, E. W. Hones, and I. B. Strong, Characteristics of the plasma sheet in the earth's magnetic tail, J. Geophys. Res., 72, 113-129, 1967.

Beard, D. B., The solar wind geomagnetic field boundary, Rev. Geophys., 2, 335$365,1964$.

Behannon, K. W., The bow shock, magnetosheath, and geomagnetic tail, Trans. Am. Geophys. Union, 48, 160, 1967; Initial mapping of the earth's bow shock, magnetosheath and magnetic tail by Explorer 33, Masters Thesis, Virginia Polytechnic Institute, Blacksburg, Virginia, May 1967. 
Behannon, K. W. and N. F. Ness, Magnetic storms in the earth's magnetic tail, J. Geophys. Res., 71, 2327-2351, 1966.

Bridge, H., S. Egidi, A. Lazarus, E. Lyon and L. Jacobson, Preliminary results of plasma measurements on IMP-A, Space Research V, 969-978, 1965.

Cahill, L. J., Inflation of the magnetosphere near 8 earth radii in the dark hemisphere, Space Research VI, 662-678, 1966.

Cahill, L. J. and D. H. Bailey, Distortion of the magnetosphere during a magnetic storm on September 30, 1961, J. Geophys. Res., 72, 159-169, 1967.

Coon, J., Vela satellite measurements of particles in the solar wind and the distant geomagnetosphere, Radiation Trapped in the Earth's Magnetic Field, ed. B. M. McCormac, D. Reidel, Dordrecht, Holland, 1966.

Dessler, A. J., Length of the magnetospheric tail, J. Geophys. Res., 69, 3913$3918,1964$.

Dungey, J. W., The length of the magnetospheric tail, J. Geophys. Res., 70, $1753,1965$.

Efroymson, M. A., Multiple regression analysis, Mathematical Methods for Digital Computers, ed A. Ralston and H. S. Wilf, John Wiley and Sons, New York, 1965.

Fairfield, D. H. and N. F. Ness, Magnetic field measurements with the IMP 2 satellite, J. Geophys. Res., 72, 2379-2403, 1967. 
Fan, C. Y., G. Gloeckler, and J. A. Simpson, Acceleration of electrons near the earth's bow shock and beyond, J. Geophys. Res., 71, 1837-1856, 1966.

Frank, L. A., A survey of electrons $\mathrm{E}>40 \mathrm{kev}$ beyond 5 earth radii with Explorer 14, J. Geophys. Res., 70, 1593-1626, 1965.

Freeman, J. W., The morphology of the electron distribution in the outer radiation zone and near the magnetospheric boundary as observed by Explorer 12, J. Geophys. Res., $69,1691-1723,1964$.

Goldman, S., Information Theory, McGraw-Hill, New York, 1953.

Gosling, J. T., J. R. Asbridge, S. J. Bame and I. B. Strong, Vela 2 measurements of the magnetopause and bow shock positions, J. Geophys. Res., $\underline{72}$, $101-112,1967$.

Gringaus, K. I., V. G. Kurt, V. I. Moroz, and I. S. Shklovsky, Results of observations of charged particles observed out to $100,000 \mathrm{~km}$ with the aid of charged particle traps on Soviet space probes, Astron. Zh., 37-4, 716-735, 1960.

Harris, C. A., AIMP-D prototype (01) and flight (02) spacecraft final deperm and AMR pre-launch mapping, NASA-GSFC Memorandum Report No. 665022 , Dec. 7, 1966.

Heppner, J. P., Recent measurement of the magnetic field in the outer magnetosphere and boundary regions, NASA-GSFC preprint X-612-65-490, November, 1965. 
Heppner, J. P., M. Campbell, T. L. Skillman, and B. G. Ledley, Preliminary report on EGO magnetic field measurements, Amer. Geophys. Union Annual Meeting, April, 1965.

Heppner, J. P., M. Sugiura, T. L. Skillman, B. G. Ledley and M. Campbell, OGO-A magnetic field observations, NASA-GSFC preprint X-612-67-150, March 1967; to be published, J. Geophys. Res.

Holtzer, R. E., M. G. McLeod, and E. J. Smith, Preliminary results from the OGO 1 search coil magnetometer: boundary positions and magnetic noise spectra, J. Geophys. Res., 프, 1481-1486, 1966.

Lincoln, J. V., Geomagnetic and solar data, J. Geophys. Res., 72, 1660, 1967. McDiarmid, I. B., and J. R. Burrows, Electron fluxes at 1000 kilometers associated with the tail of the magnetosphere, J. Geophys. Res., 70, 3031$3044,1965$.

Montgomery, M. D., S. Singer, J. P. Conner, and E. E. Stogsdill, Spatial distribution, energy spectra, and time variations of energetic electrons $(E>50$ kev) at 17.7 earth radii, Phys. Rev. Letters, 14, 209-213, 1965.

Ness, N. F., The earth's magnetic tail, J. Geophys. Res., 70, 2989-3005, 1965.

Ness, N. F., Observations of the solar wind interaction with the geomagnetic field: conditions quiet, NASA-GSFC preprint X-612-66-381, August 1966; to be published in Proceedings of Belgrade Solar Terrestrial Relationships Interunion Meeting, 1966. 
Ness, N. F., K. W. Behannon, S. C. Cantarano and C. S. Scearce, Observations of the earth's magnetic tail and neutral sheet at 510,000 kilometers by Explorer 33, J. Geophys. Res., 72, 927-933, 1967a.

Ness, N. F., C. S. Scearce and J. B. Seek, Initial results of the IMP 1 magnetic field experiment, J. Geophys. Res., 69, 3531-3570, 1964.

Ness, N. F., C. S. Scearce, J. B. Seek and J. M. Wilcox, Summary of results from the IMP 1 magnetic field experiment, Space Research VI, 581-628, 1966.

Ness, N. F., C. S. Scearce and S. Cantarano, Probable observation of the geomagnetic tail at $6 \times 10^{6}$ kilometers by Pioneer 7 , NASA-GSFC preprint X-612-67-183, April, 1967b.

Ness, N. F. and D. J. Williams, Correlated magnetic tail and radiation belt observations, J. Geophys. Res., 71, 322-325, 1966.

Serbu, G. P., Results from the IMP 1 retarding potential analyzer, Space Research V, 564-574, 1965.

Serbu, G. P. and E. J. R. Maier, Low energy electrons measured on IMP 2, J. Geophys. Res., 71, 3755-3766, 1966.

Singer, S., J. P. Conner, W. D. Evans, M. D. Montgomery and E. E. Stogsdill, Plasma observations at $10^{5} \mathrm{~km}$, Space Research V, 546-563, 1965.

Smith, E. J. and C. P. Sonett, Satellite observations of the distant field during magnetic storms: Explorer 6, Proc. Interm. Conf. Cosmic Rays Earth Strom, Kyoto, Sept. 1961, J. Phys. Soc. Japan, 17, 17, 1962. 
Smith, E. J., C. P. Sonett, and J. W. Dungey, Satellite observations of the geomagnetic field during magnetic storms, J. Geophys. Res., 69, 2669-2688, 1964.

Speiser, T. W. and N. F. Ness, The neutral sheet in the geomagnetic tail: its motion, equivalent currents, and field line connection through it, J. Geophys. Res., 72, 131-142, 1967.

Van Allen, J. A., Absence of 40-kev electrons in the earth's magnetospheric tail at 3300 earth radii, J. Geophys. Res., 70, 4731-4739, 1965.

Van Allen, J. A. and N. F. Ness, Observed particle effects of an interplanetary shock wave on July 8, 1966, J. Geophys. Res., 72, 935-942, 1967.

Walters, G. K., Effect of oblique interplanetary magnetic field on shape and behavior of the magnetosphere, J. Geophys. Res., 69, 1769-1783, 1964.

Wolfe, J. H., R. W. Silva, and M. A. Myers, Observations of the solar wind during the flight of IMP 1, J. Geophys. Res., 71, 1319-1340, 1966. 


\section{FIGURE CAPTIONS}

Figure 1. Explorer 33 spacecraft in flight configuration with booms and solar paddles fully deployed.

Figure 2. Variation of Explorer 33 spin rate and spin axis and orbit orientation parameters during the period July 1-November 11, 1966.

Figure 3. Ecliptic plane view of the first eight orbits of Explorer 33. Distance units are earth radii. Decimal day numbers are shown.

Figure 4. Illustration of the variation of the Explorer 33 apogee position relative to the ecliptic plane due to perturbations of the orbit by the moon.

Figure 5. Magnetometer sensor frequency response and instrumental phase shift. Since the instrumental phase shift is not entirely negligible over the range of spin frequencies of the spacecraft $(0.38-0.45 \mathrm{cps})$, it is compensated for the analysis.

Figure 6. Block diagram of GSFC magnetic field experiment on Explorer 33 .

Figure 7. Example of magnetometer sensor in-flight sensitivity calibration results, showing change in sensor output in engineering units as a function of average ambient field component along the sensor axis when a 10 gamma calibration field is applied.

Figure 8. Magnetic field data from October 4, 1966 illustrating distant multiple traversals of the dusk flank of the bow shock. AIMP-D is an alternate designation for Explorer 33. Field magnitude $\mathrm{F}$ and magnitude rootmean-square deviation RMS are in units of gammas. 
Figure 9. Magnetic field data from October 19, 1966 illustrating magnetosheath fields and multiple traversals of the tail magnetopause. Note that at noon the spacecraft was approximately $34 R_{E}$ from the earth-sun line.

Figure 10. Summary of Explorer 33 bow shock and magnetopause traversals (rotated into ecliptic plane), illustrating both the variability in the instantaneous position of the boundaries and the apparent asymmetry of the average boundaries about the earth-sun line.

Figure 11. View from the earth of the downstream segments of orbits 1-8 and the cross section of a cylindrical magnetic tail.

Figure 12. Explorer 33 bow shock and magnetopause traversals in solar magnetospheric coordinates for four distance intervals down the tail revealing the noncylindrical shape of the magnetic tail.

Figure 13. Average vector magnetic field topology in the geomagnetic tail during July-November 1966, as typified by the projection of hourly average field vectors onto the plane perpendicular to the solar magnetospheric equatorial plane.

Figure 14. $\mathrm{X}_{\mathrm{SM}} \mathrm{Y}_{\mathrm{SM}}$-plane projection of the hourly average tail field vectors observed by Explorer 33 during July-November 1966 at distances $Z_{S M} \geq 3 R_{E}$ above the solar magnetospheric equatorial plane.

Figure 15. $\mathrm{X}_{\mathrm{SM}} \mathrm{Y}_{\mathrm{SM}}$-plane projection of the hourly average tail field vectors observed by Explorer 33 at $Z_{S M}<3 R_{E}$. 
Figure 16. Magnetic field data from October 21, 1966 illustrating the steady character of the undisturbed magnetic tail field. These data are typical of all quiet day (low $\mathrm{Kp}$ ) geomagnetic tail field observations by Explorer 33 .

Figure 17. Regular-run magnetic observatory horizontal component magnetograms for the September 14, 1966 sudden commencement storm period.

Figure 18. Solar ecliptic and midnight meridian plane views of orbit 5 . The dashed lines indicate the interval during which the magnetopause was observed by the spacecraft as it entered the tail.

Figure 19. Magnetic field data from September 14, 1966 illustrating the commencement of the September 14, 1966 magnetic storm in the geomagnetic tail beyond the lunar orbital distance.

Figure 20. Magnetic field data from September 15, 1966 showing the recovery phase in the magnetic tail of the September 14 sudden commencement geomagnetic storm. Both high and low frequency magnetic fluctuations continued throughout that day.

Figure 21. Detailed magnetic field data from August 23, 1966 illustrating a thin neutral sheet.

Figure 22. Sequence average data showing the variations in SM field components during the August 23 neutral sheet traversal.

Figure 23. Detailed magnetic field data from August 24, 1966 illustrating an inflated neutral sheet. 
Figure 24. Sequence average data showing the variations in SM field components during the August 24 neutral sheet traversal.

Figure 25. Distribution of undisturbed hourly average magnetic tail field magnitudes as a function of geocentric distance down the tail for the period July-November 1966. 


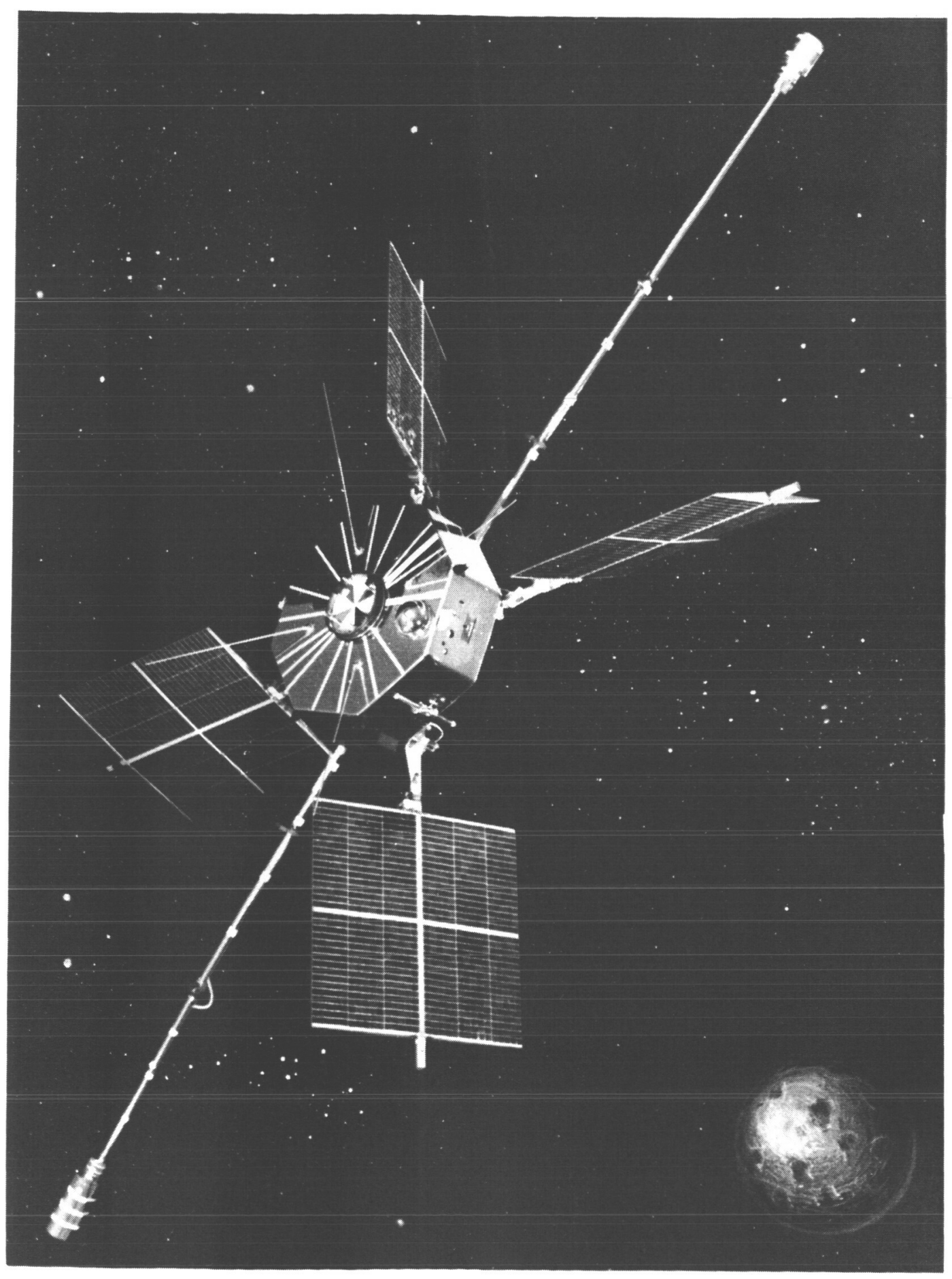

ํํㄴ 


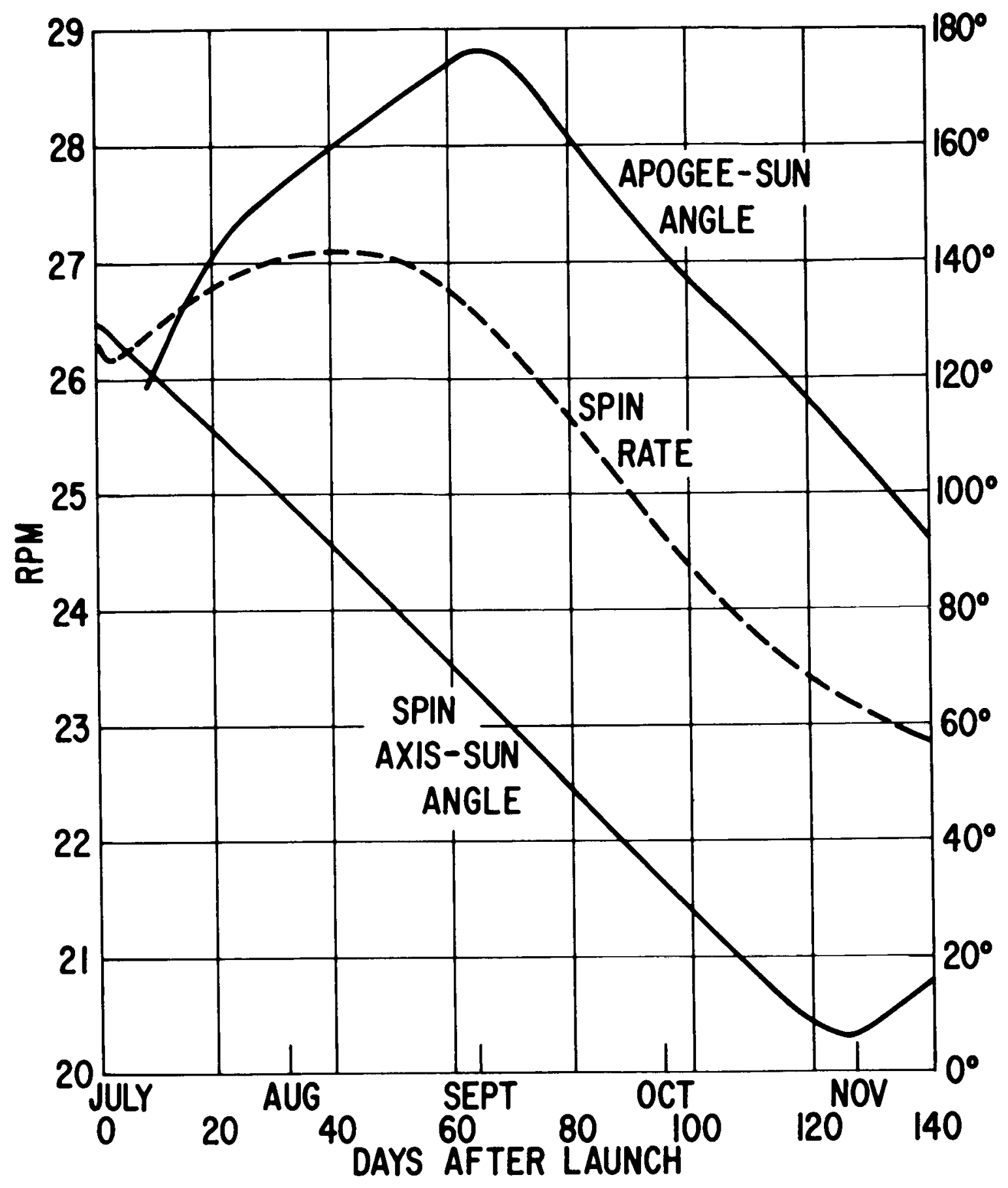

Figure 2 


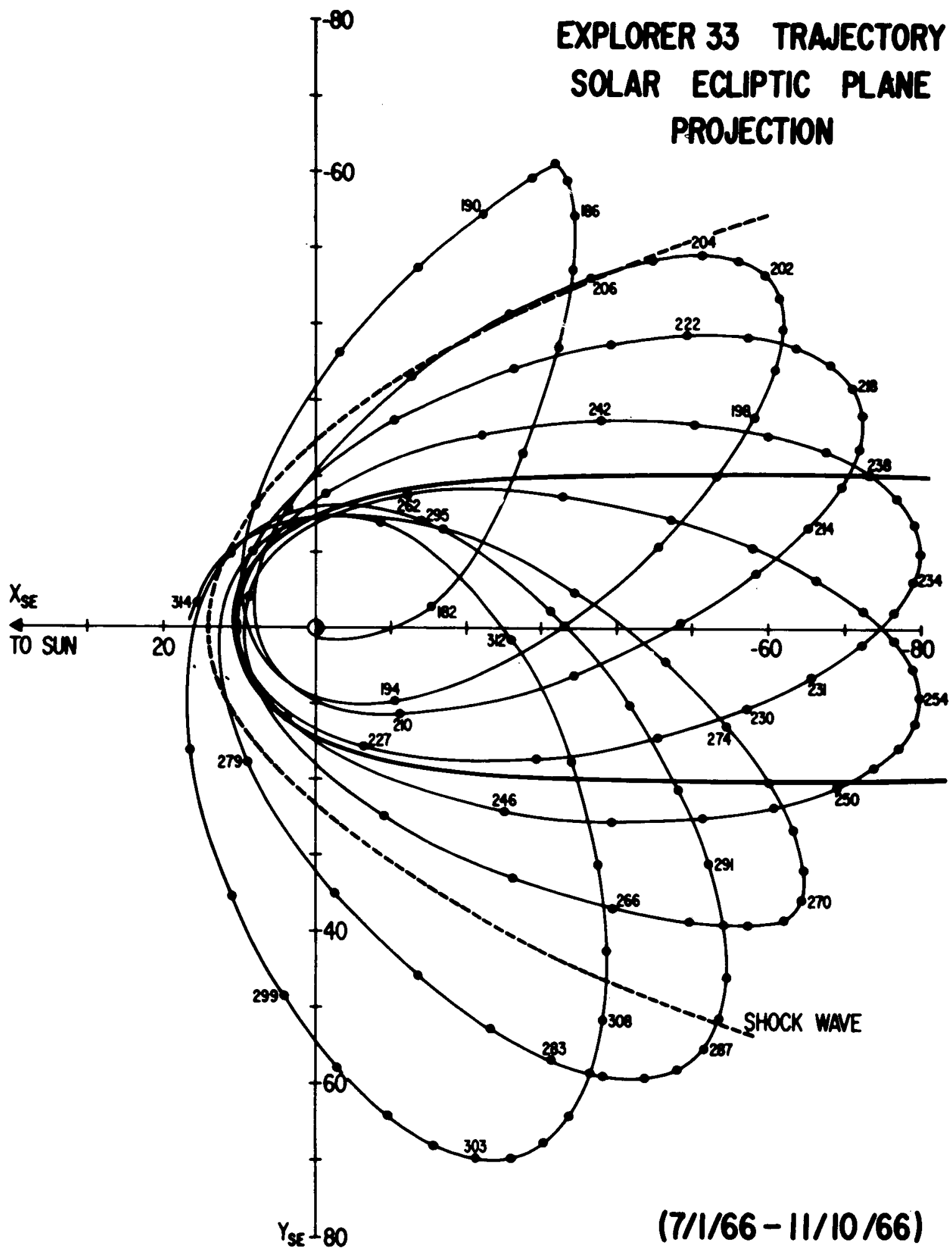

Figure 3 


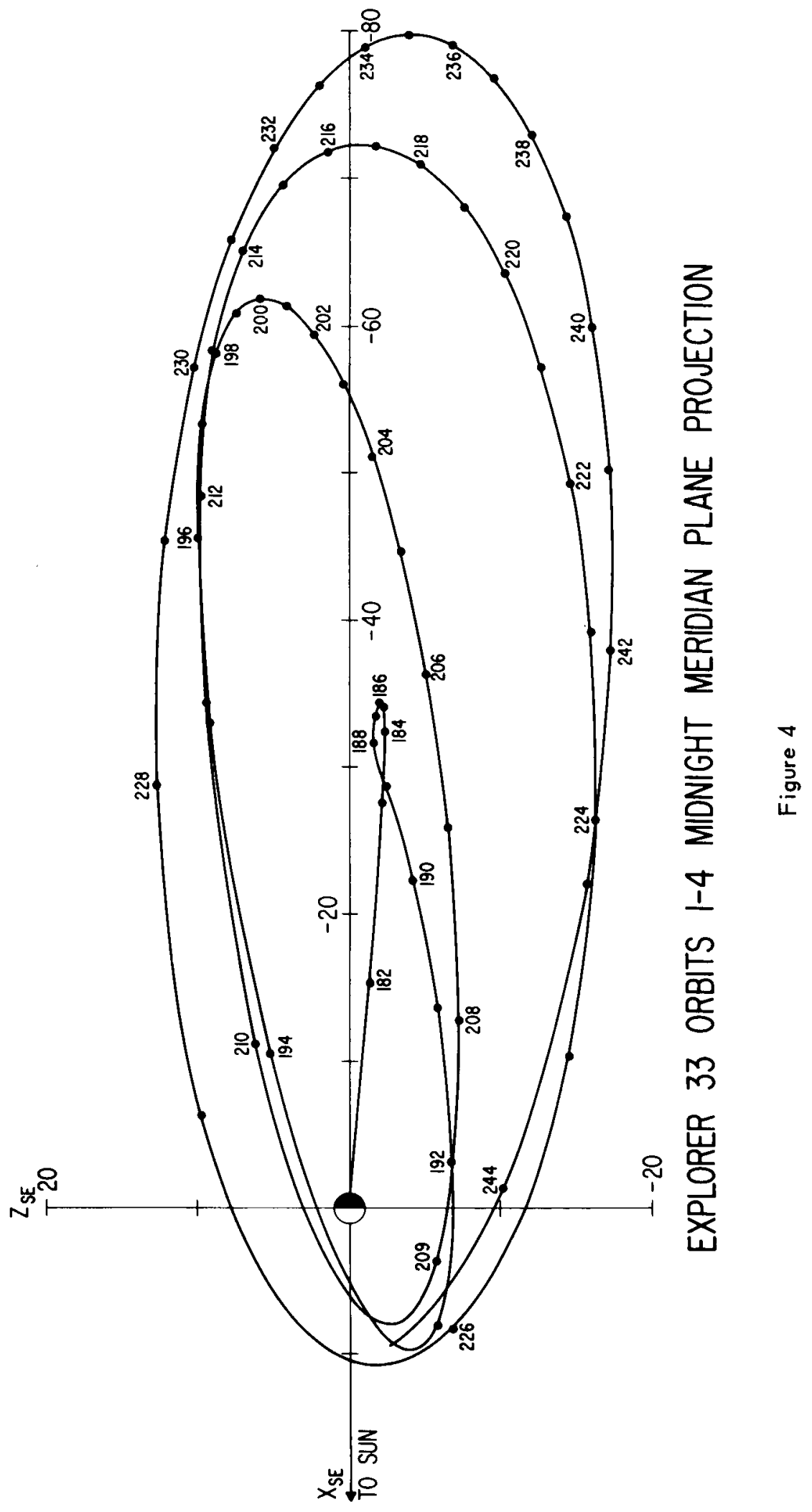




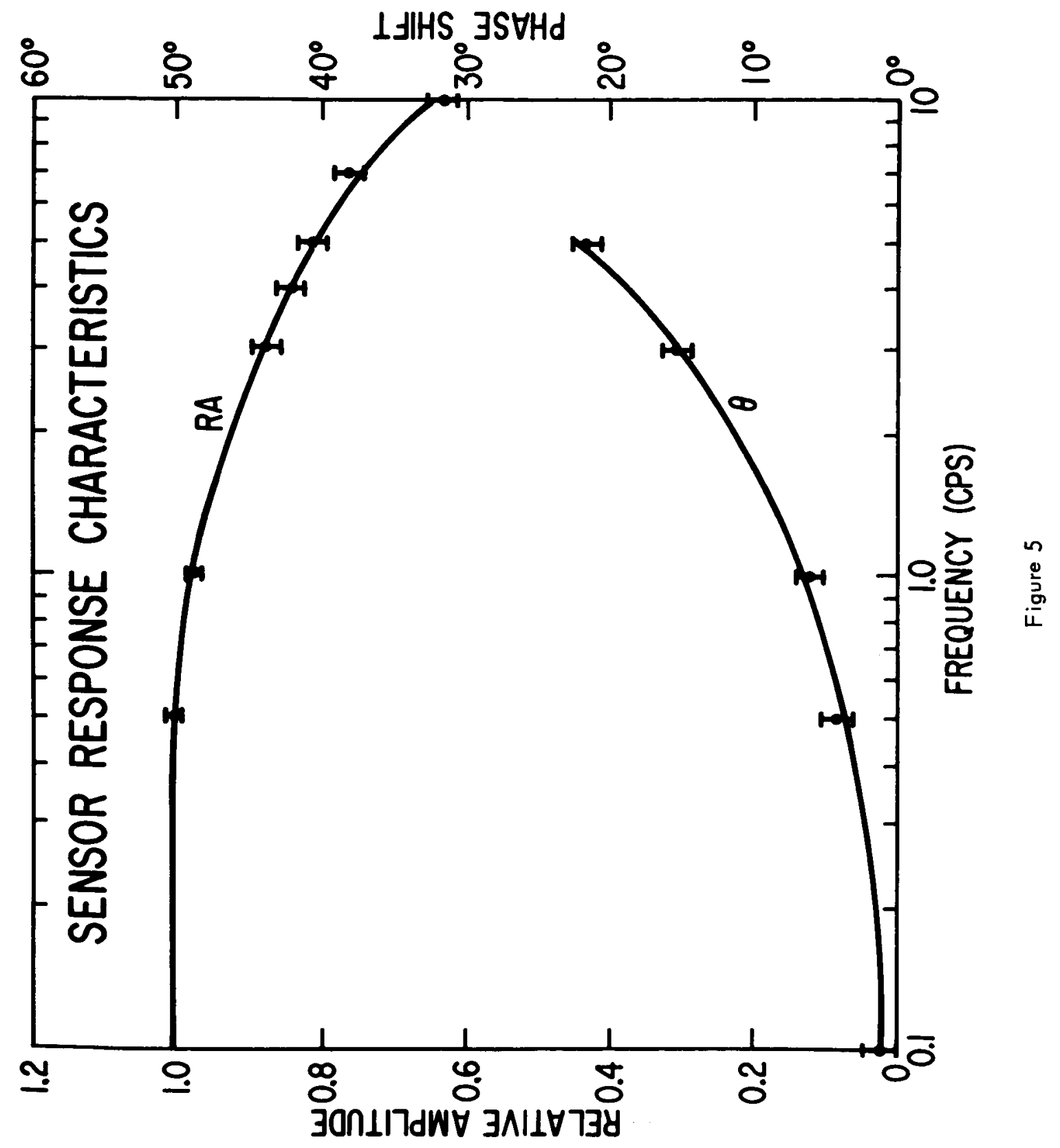




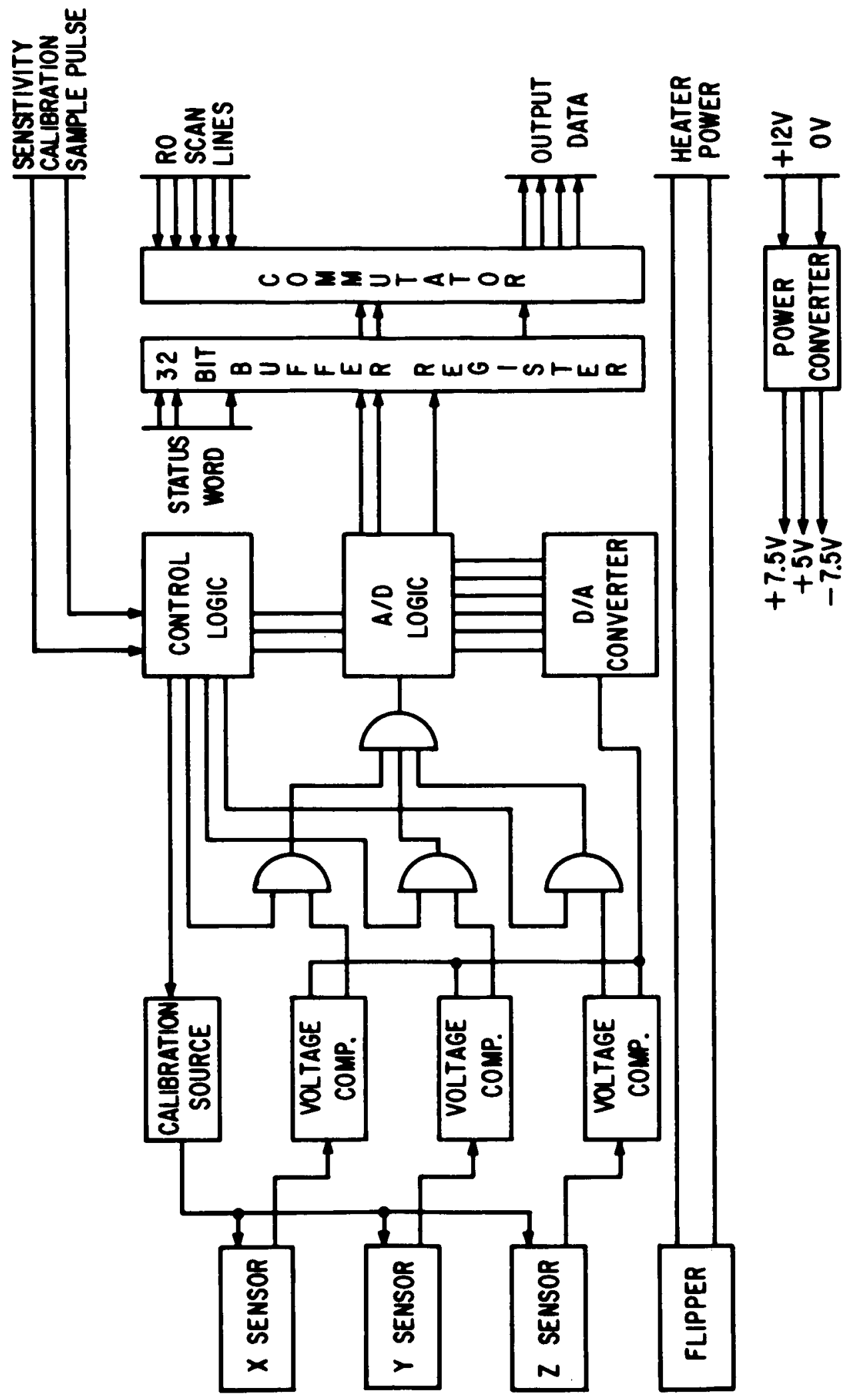

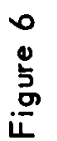




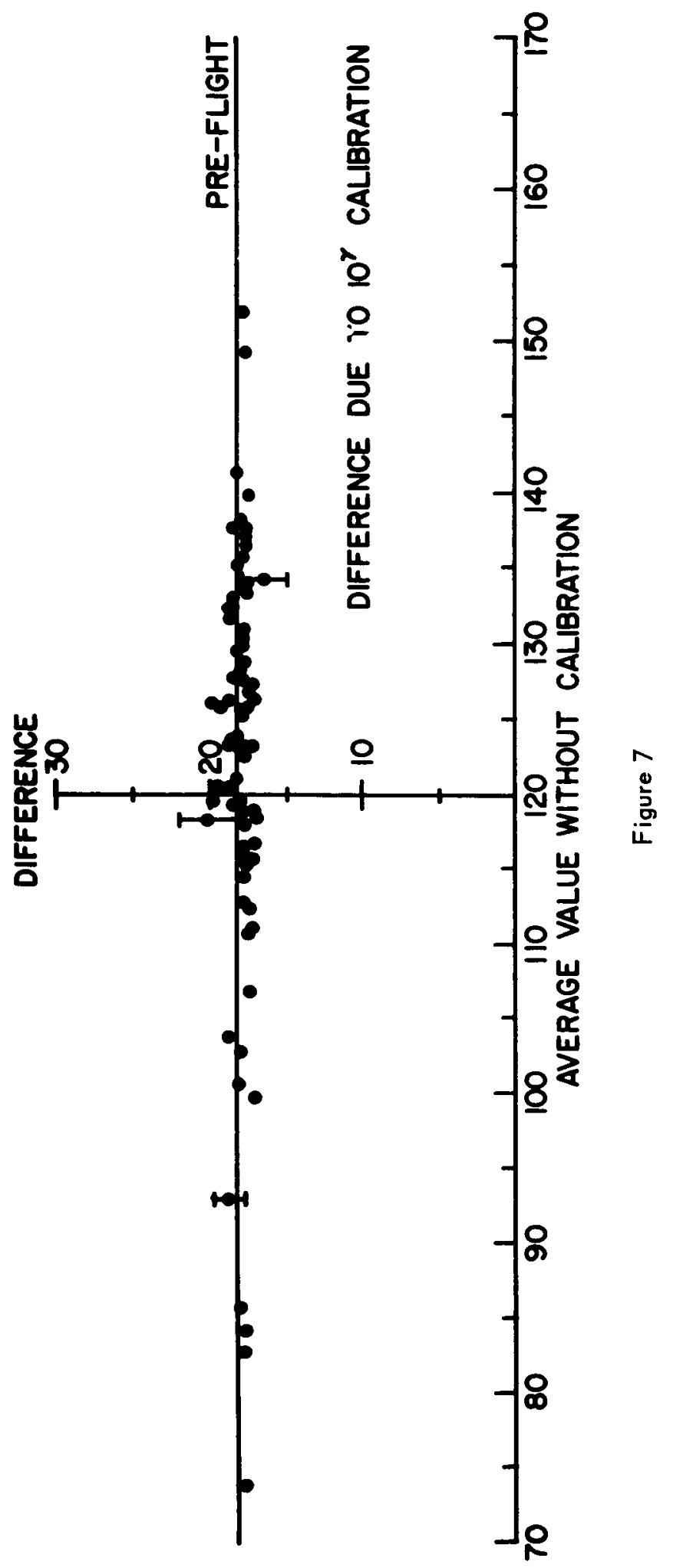



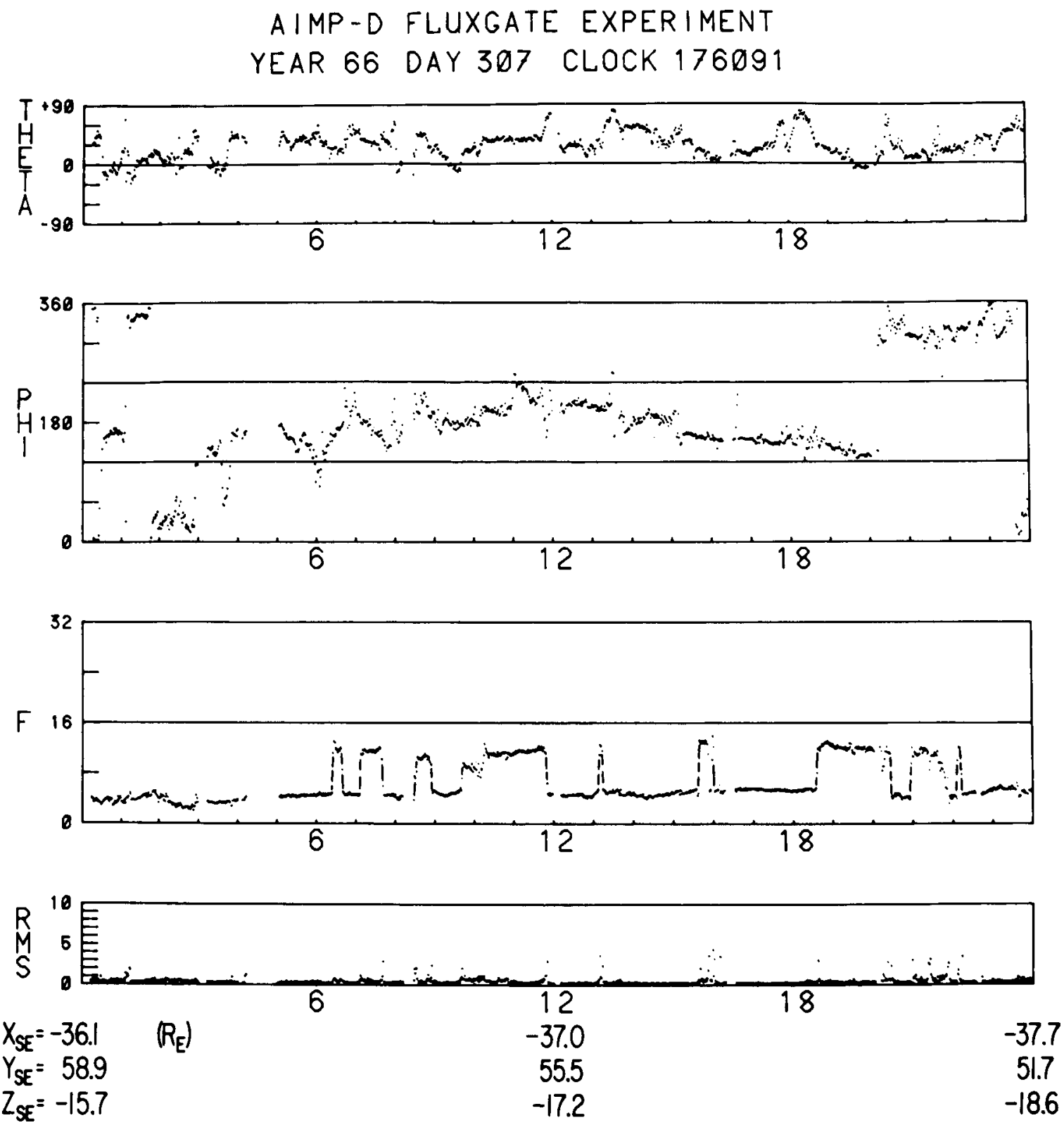

Figure 8 


\section{AIMP-D FLUXGATE EXPERIMENT \\ YEAR 66 DAY 291 CLOCK 159183}
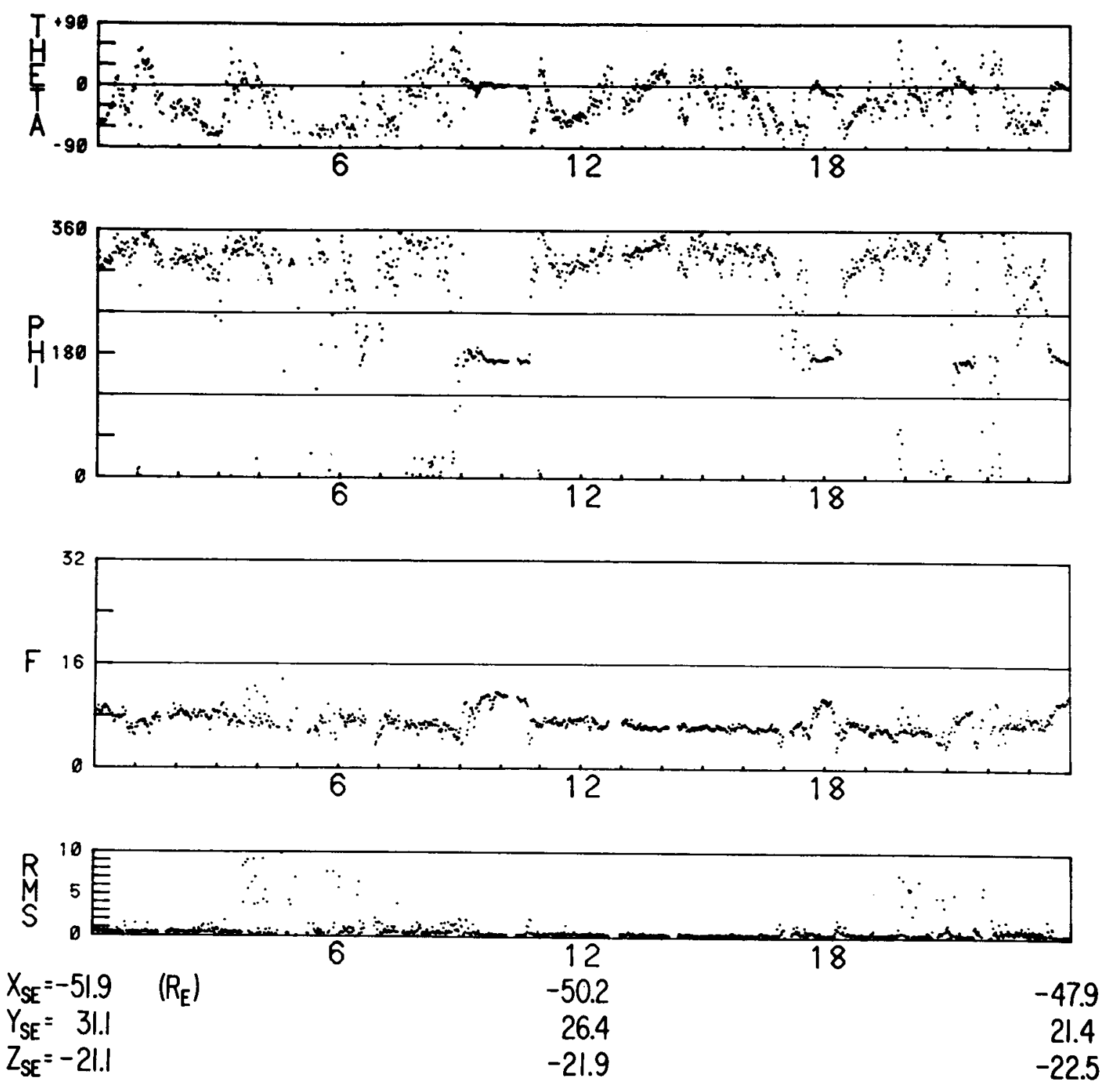

Figure 9 


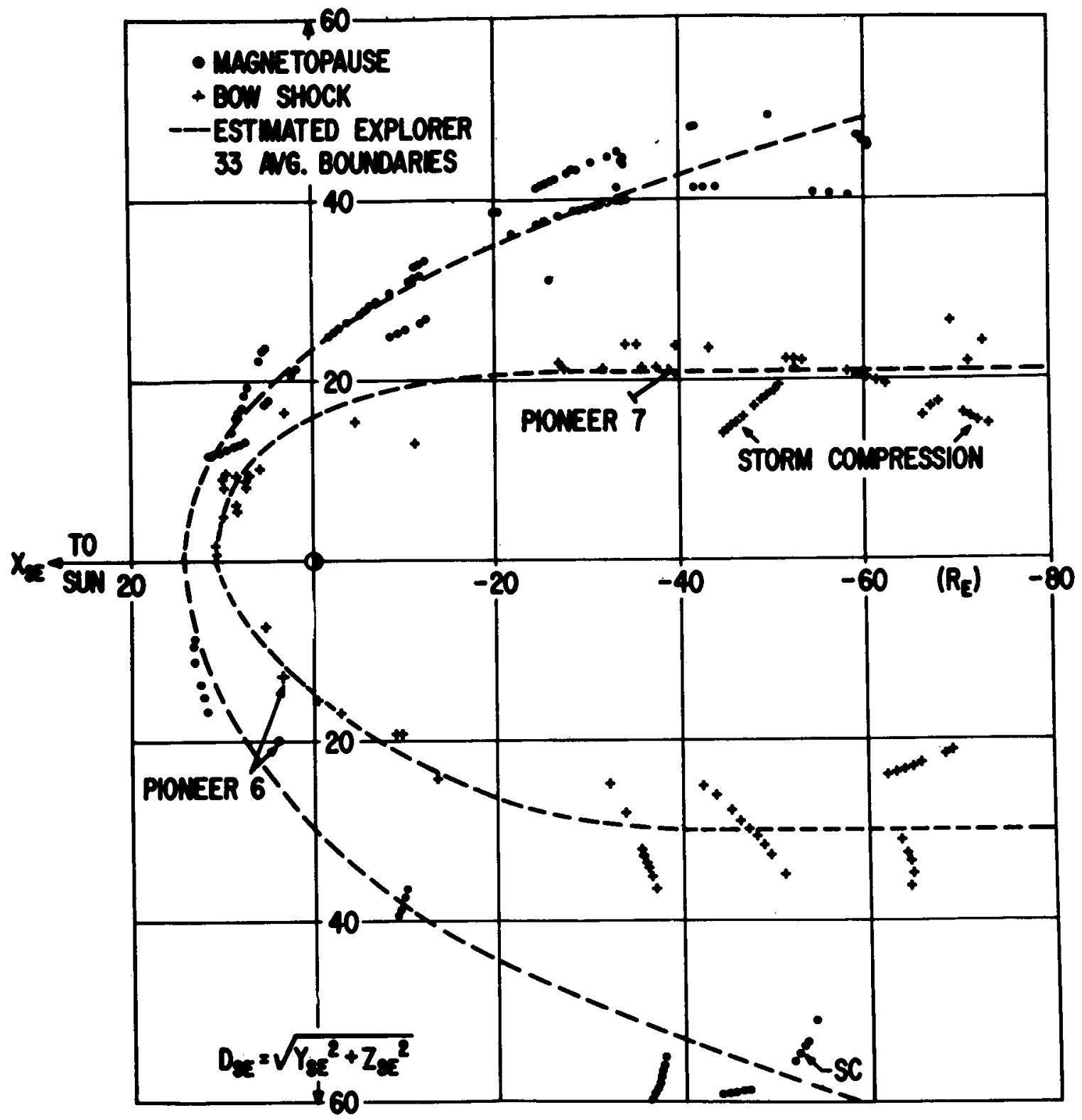

Figure 10 


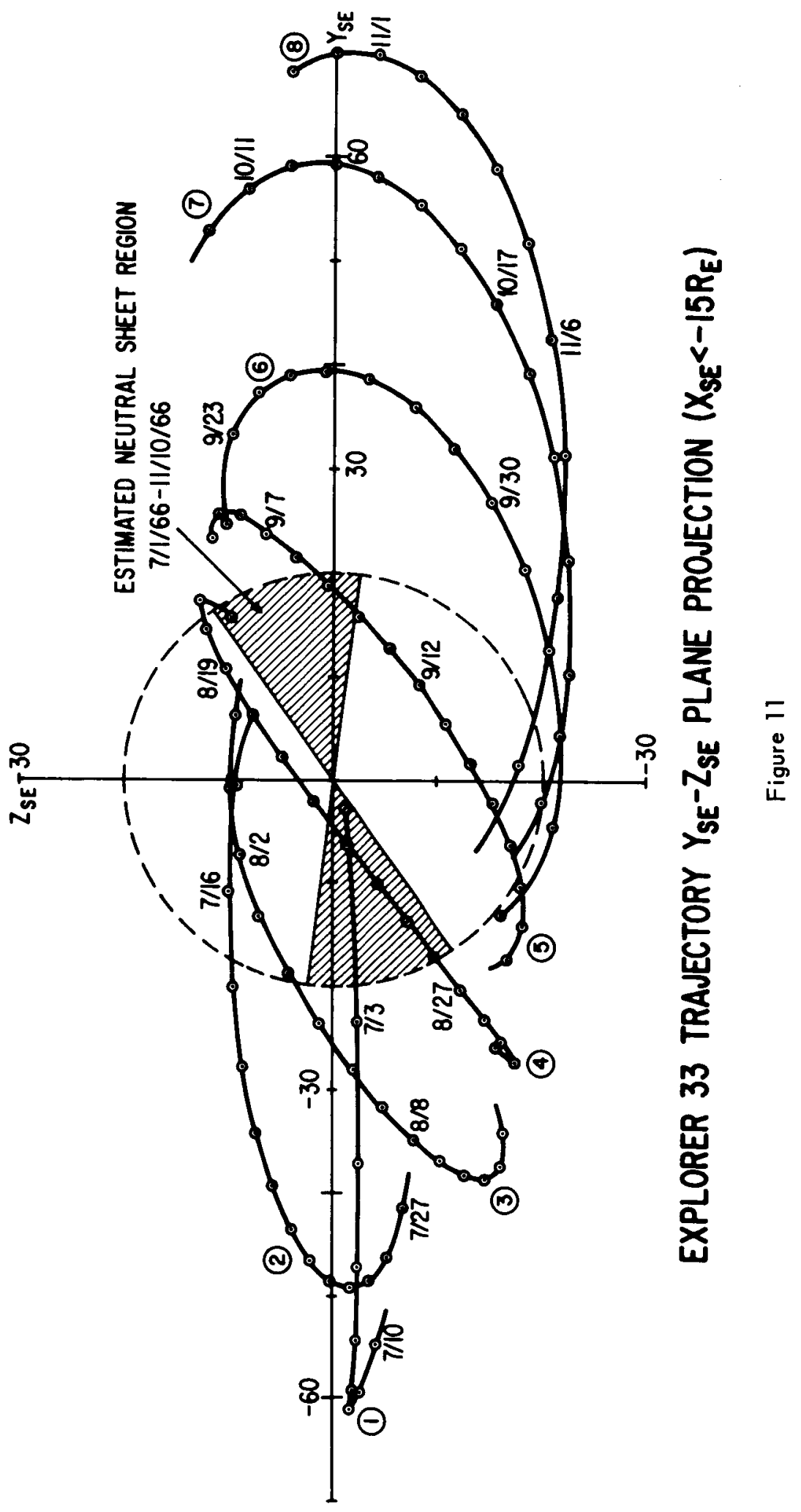



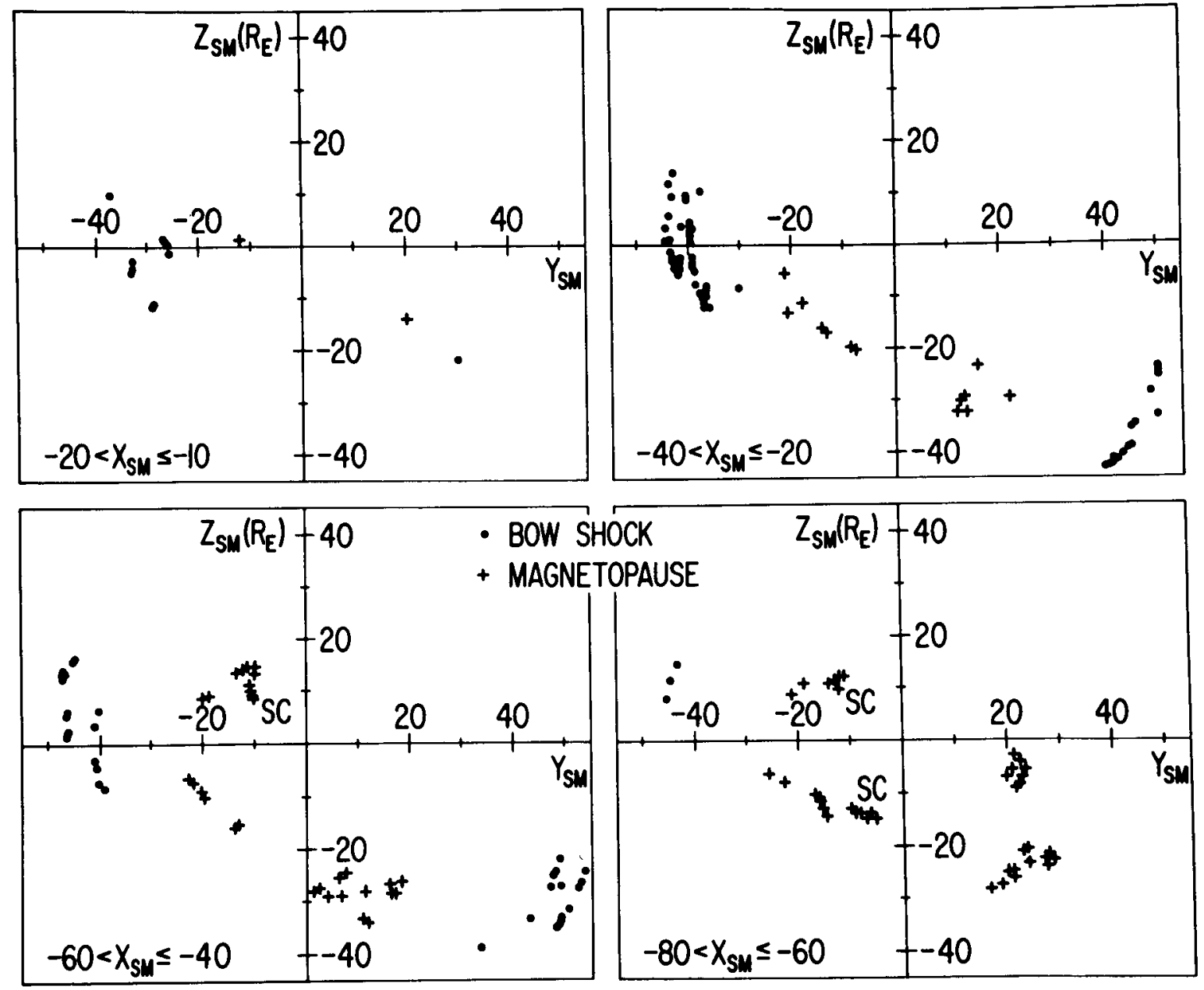

Figure 12 


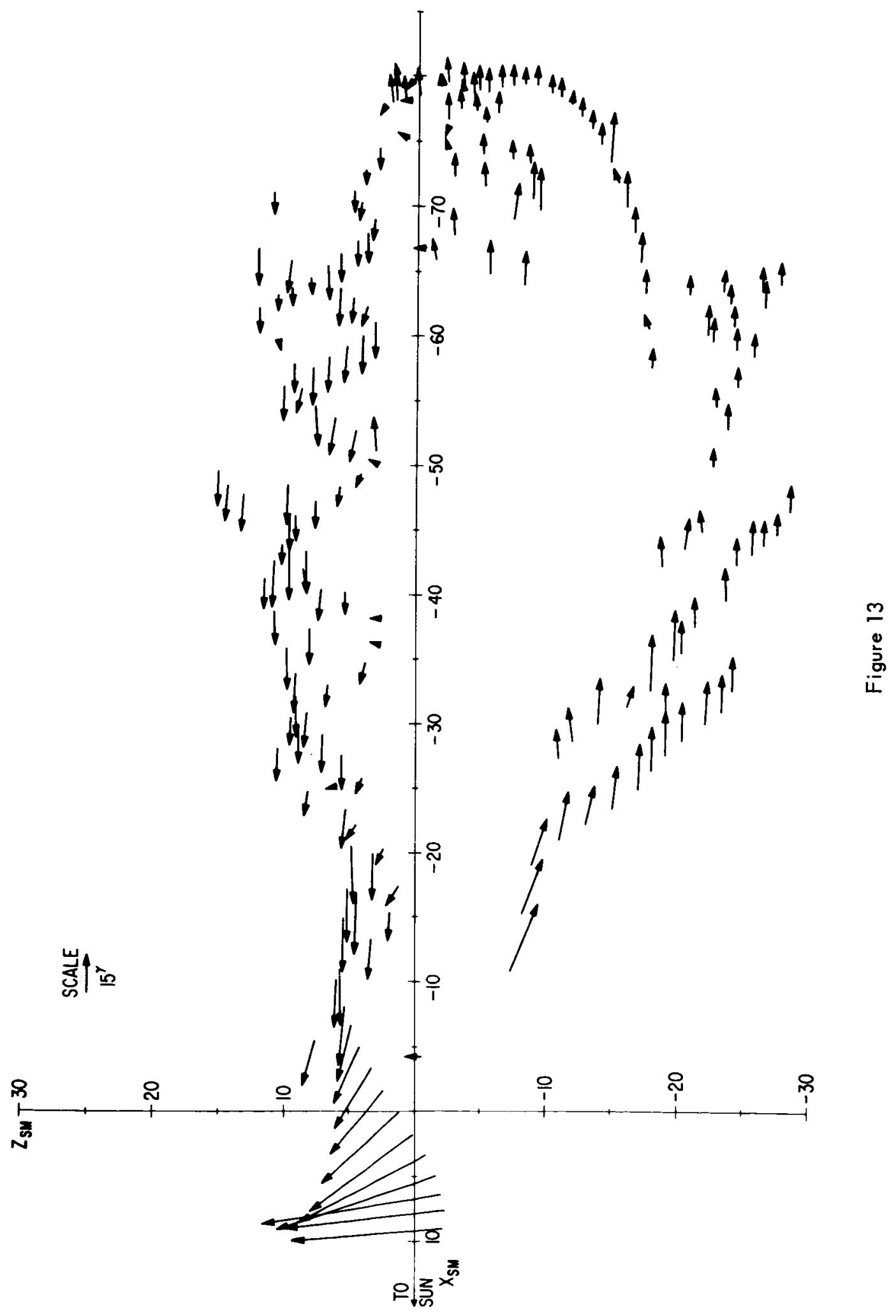




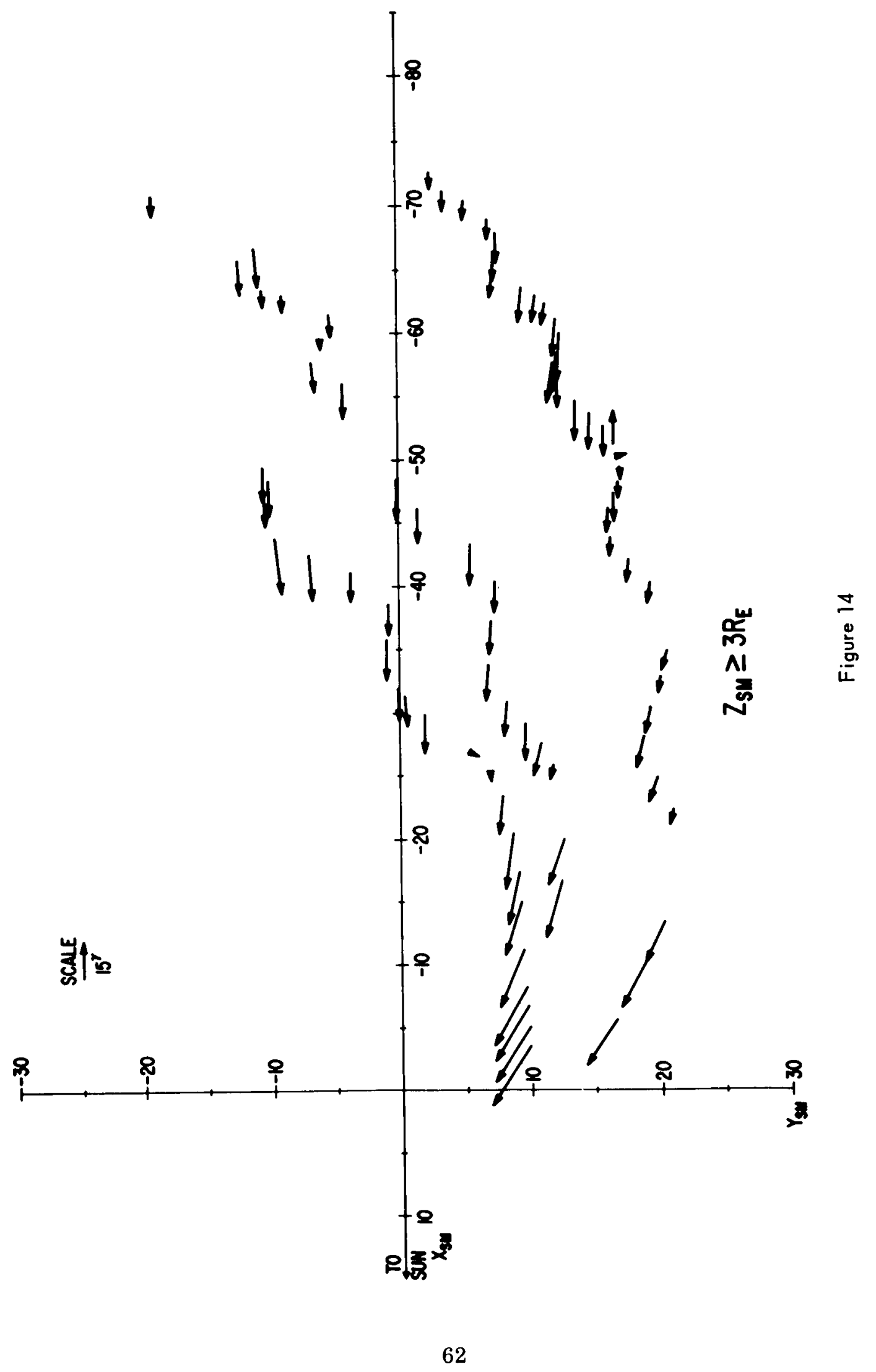




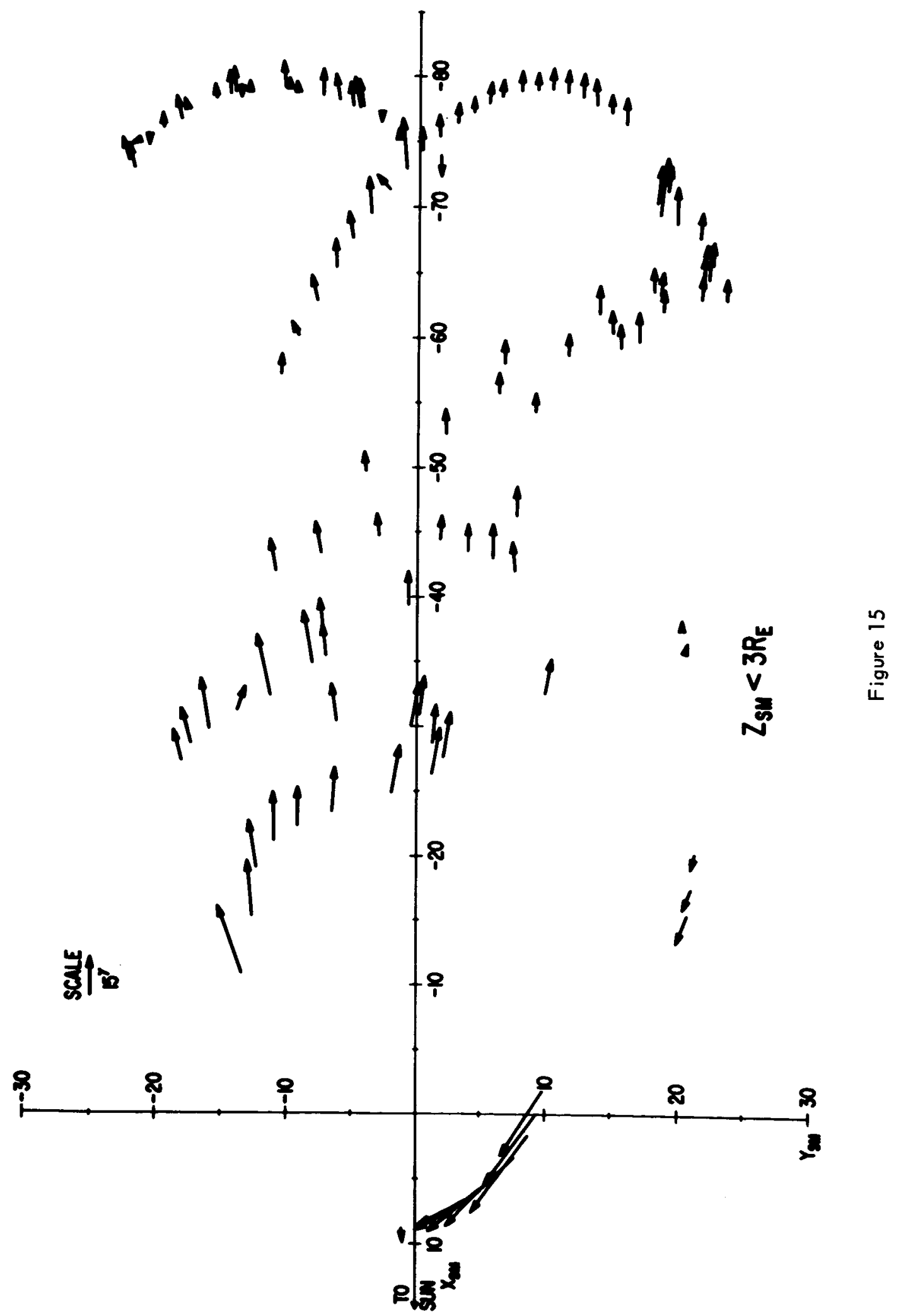


AIMP-D FLUXGATE EXPERIMENT

YEAR 66 DAY 293 CLOCK 161296
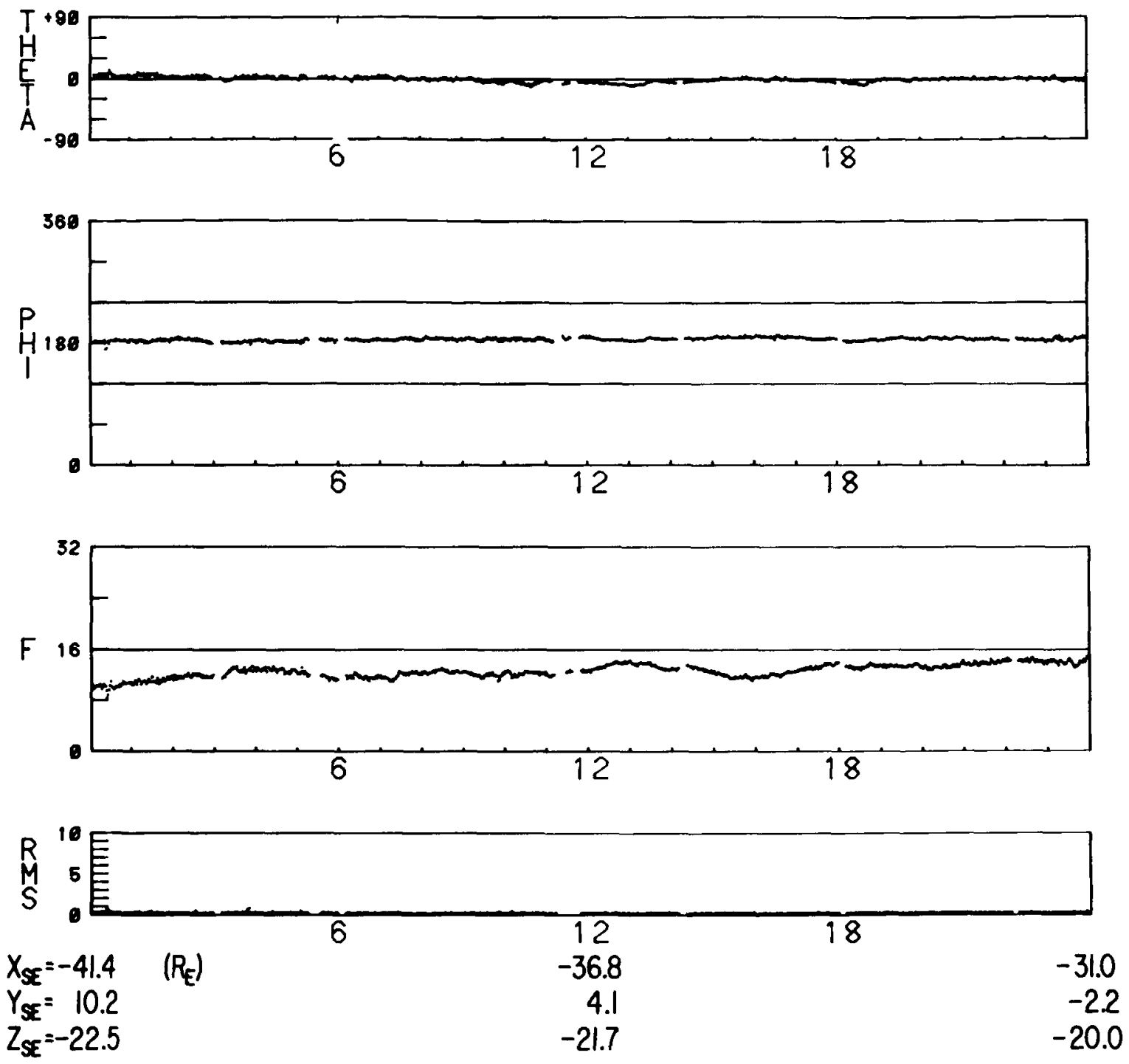

Figure 16 


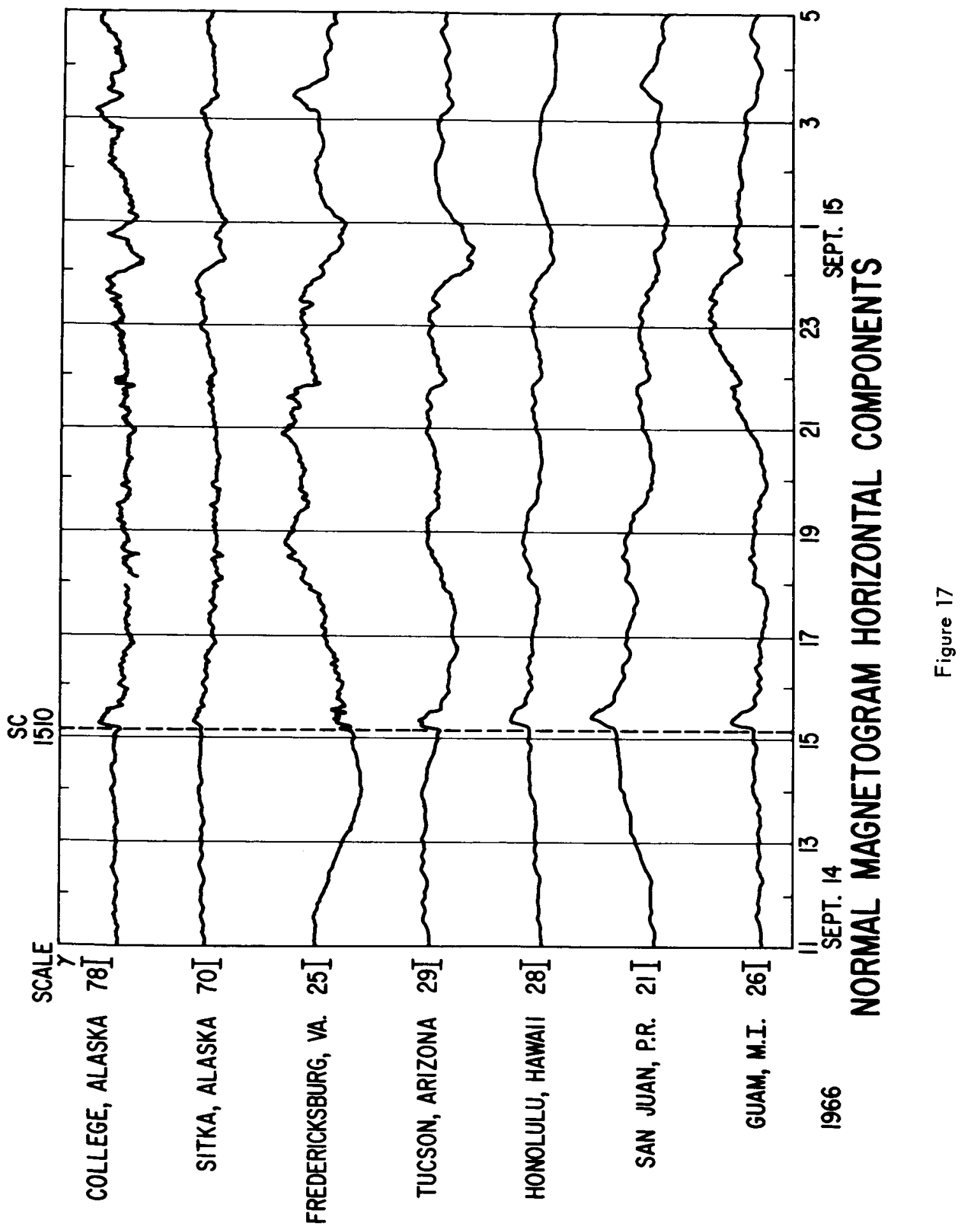



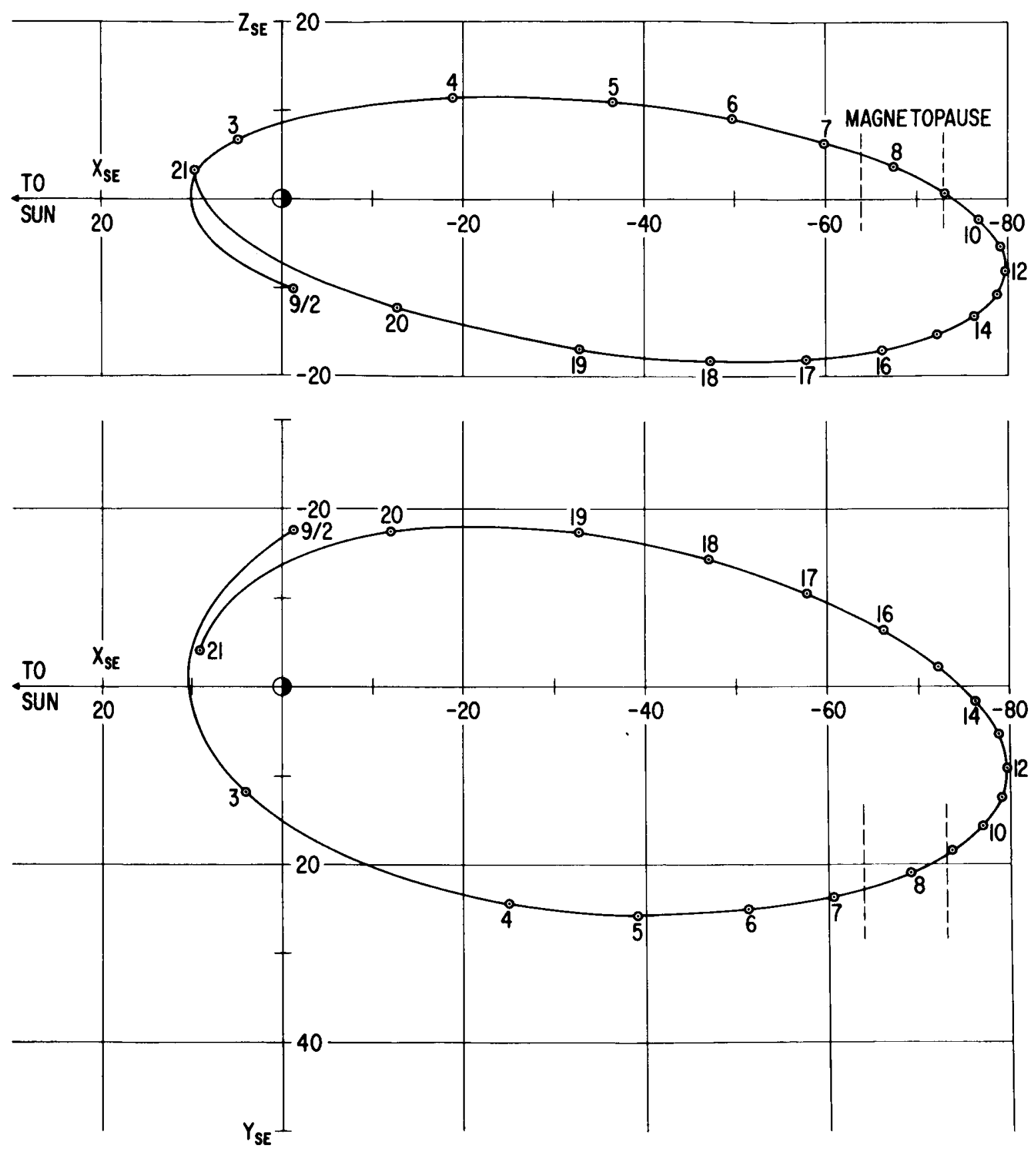

EXPLORER 33 ORBIT 5 SEPT. 1966

Figure 18 


\section{AIMP-D FLUXGATE EXPERIMENT}

YEAR 66 DAY 256 CLOCK 122219
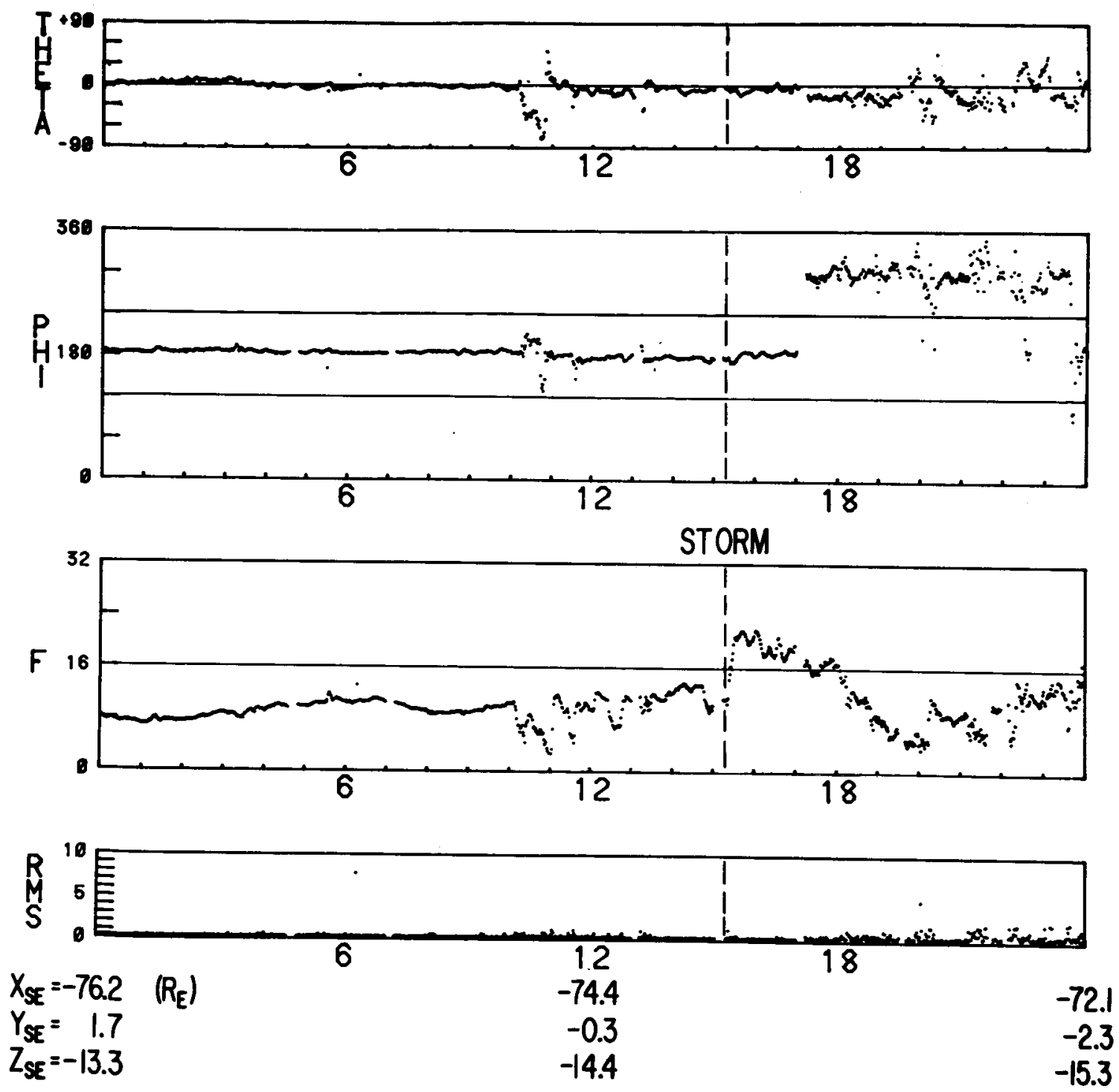

Figure 19 

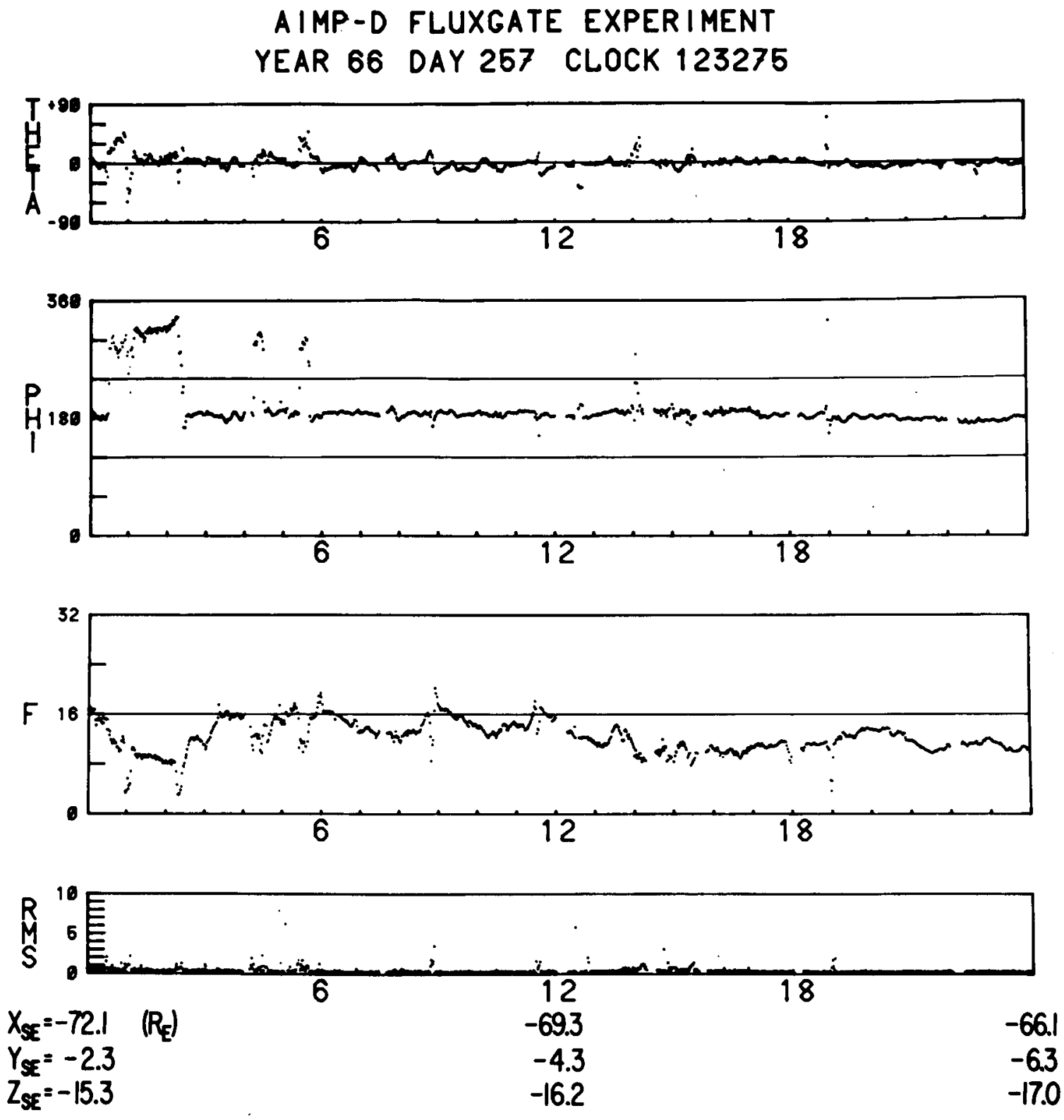

Figure 20 

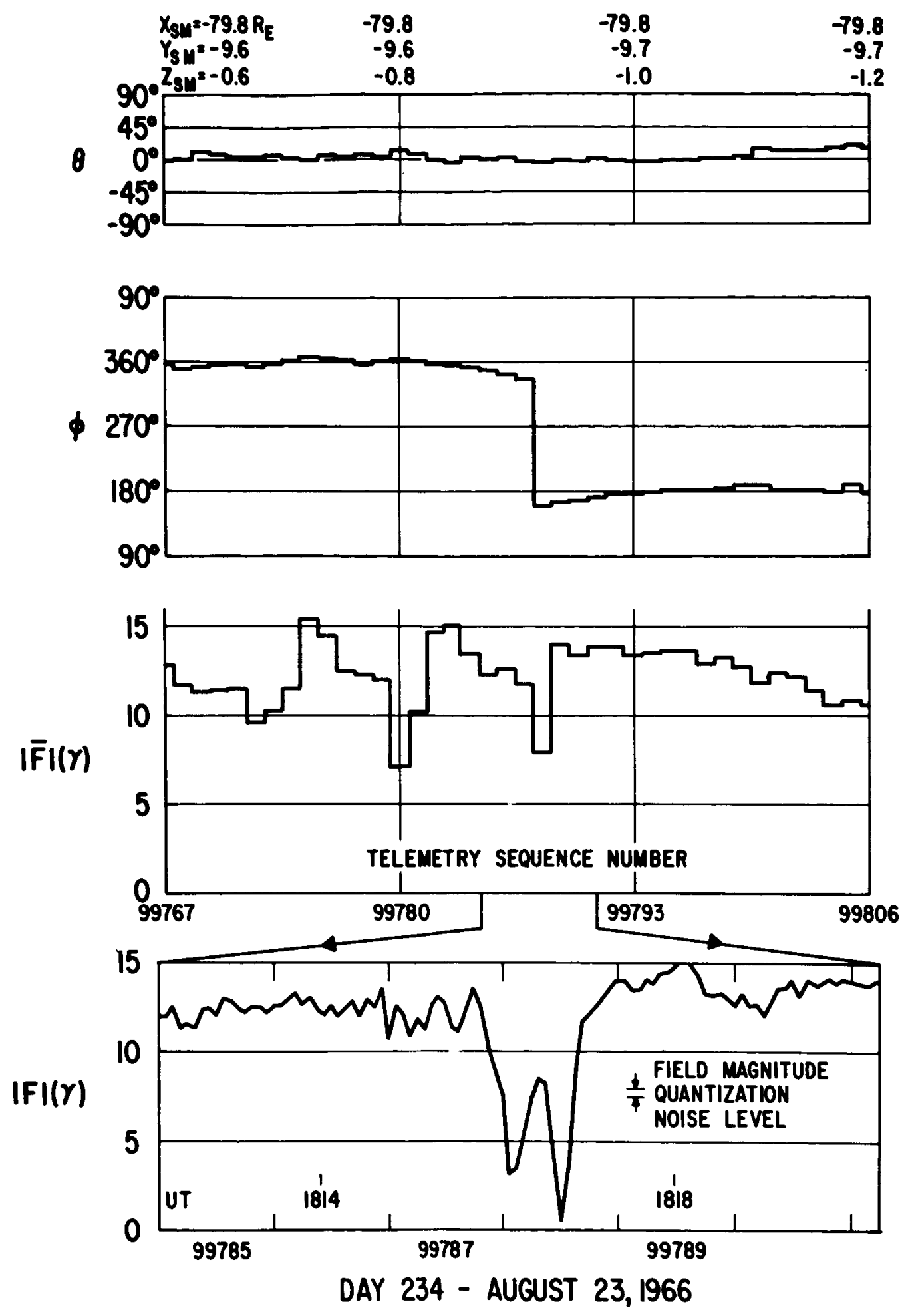

Figure 21 

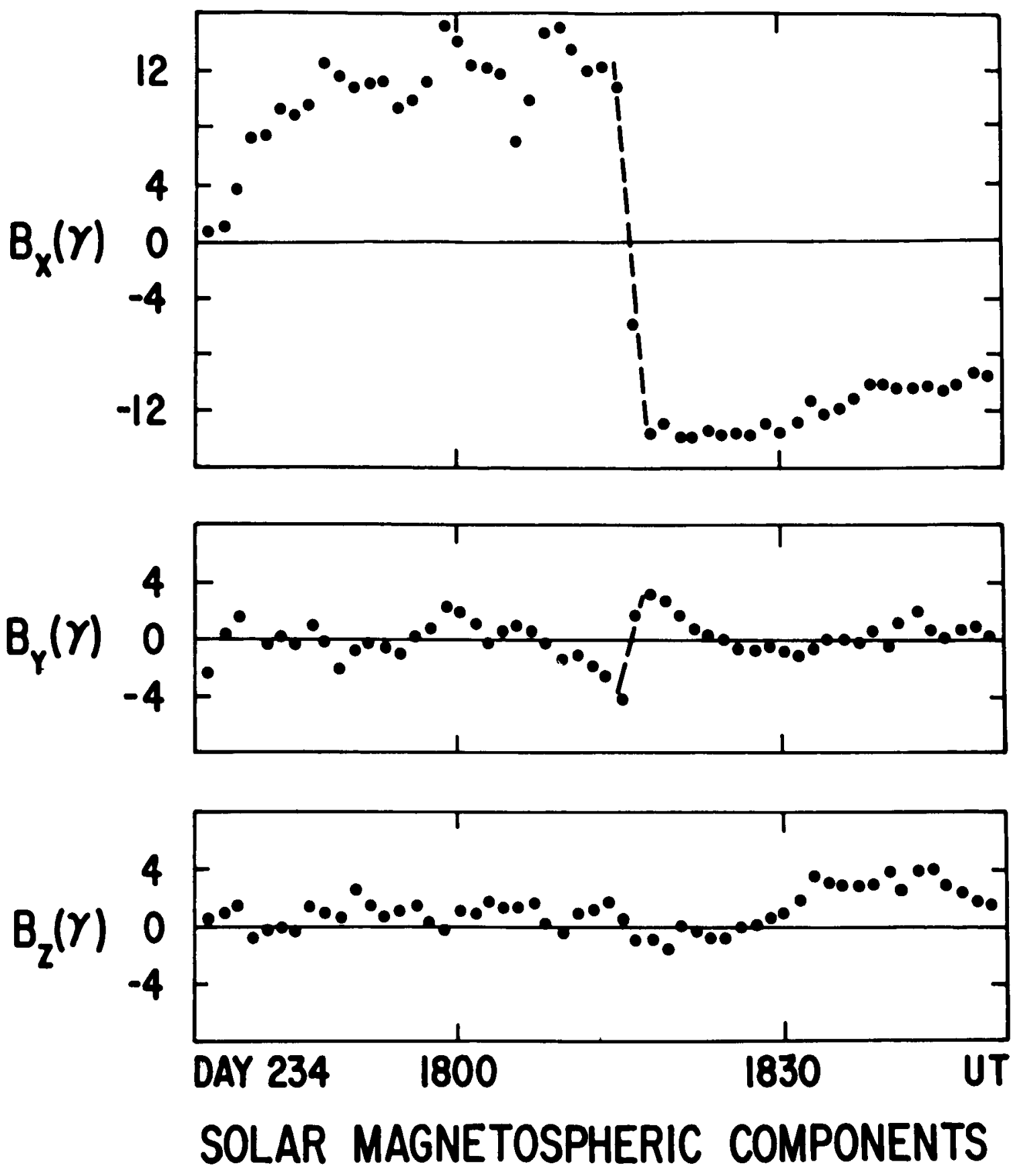

Figure 22 

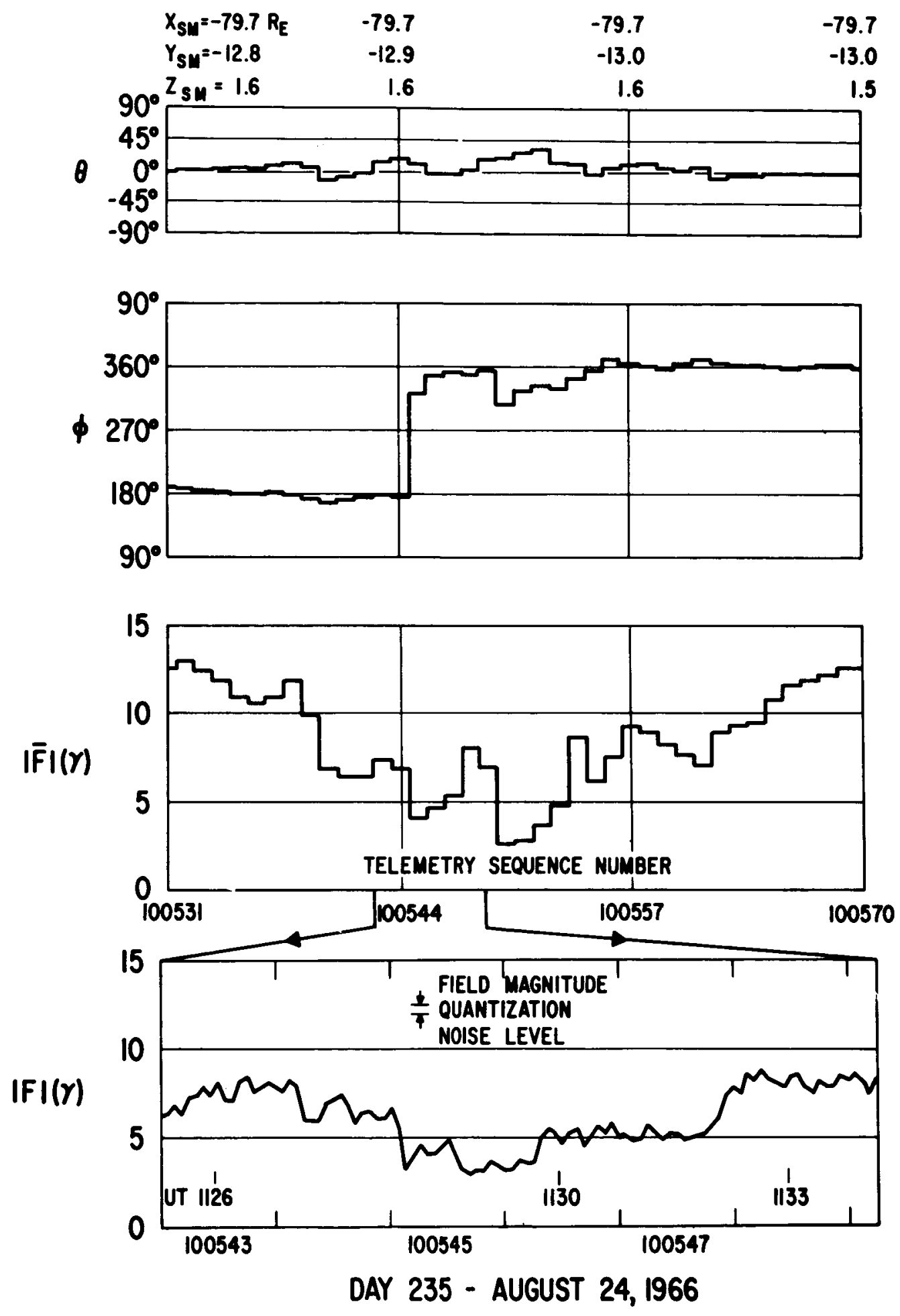

Figure 23 

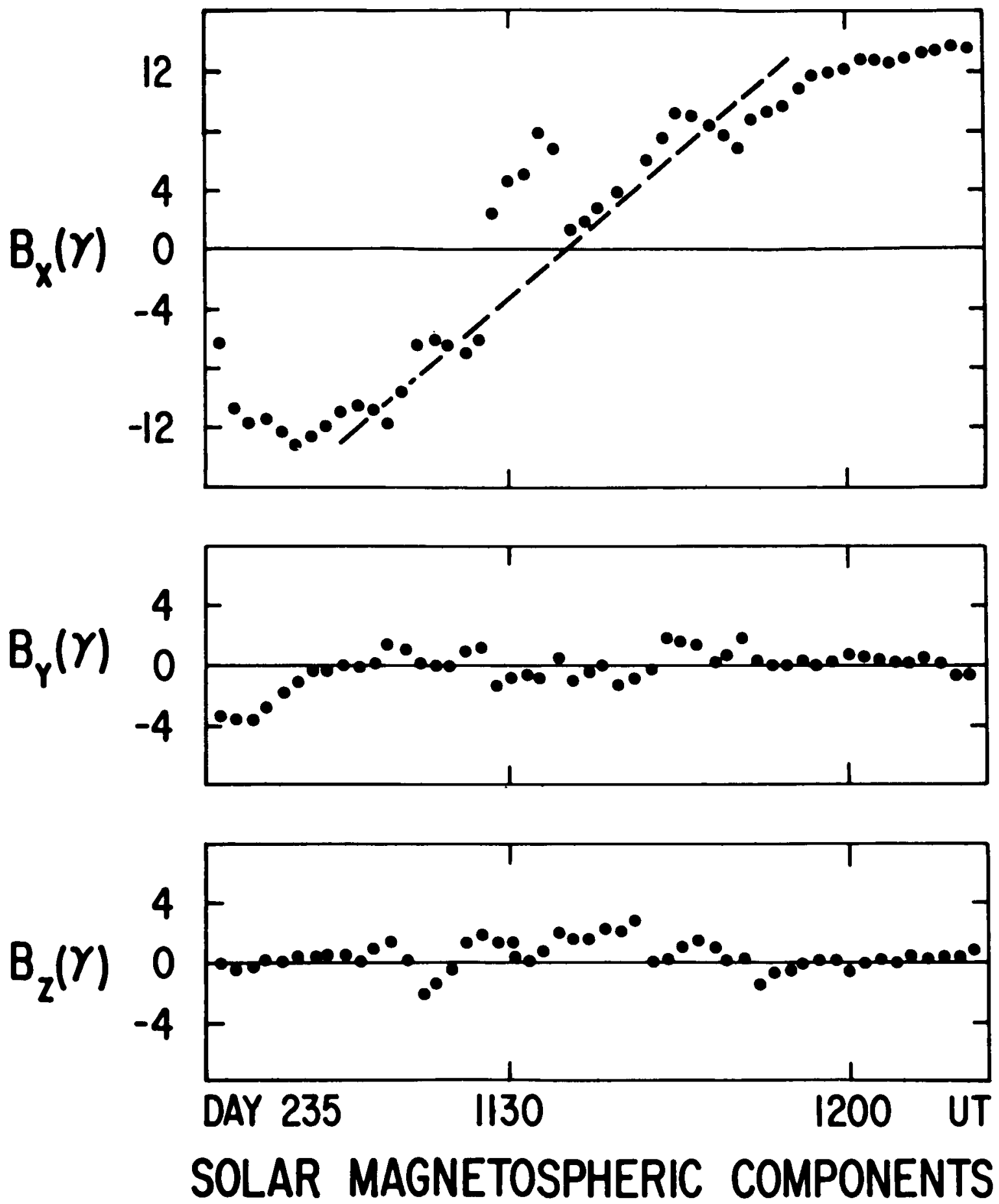

Figure 24 


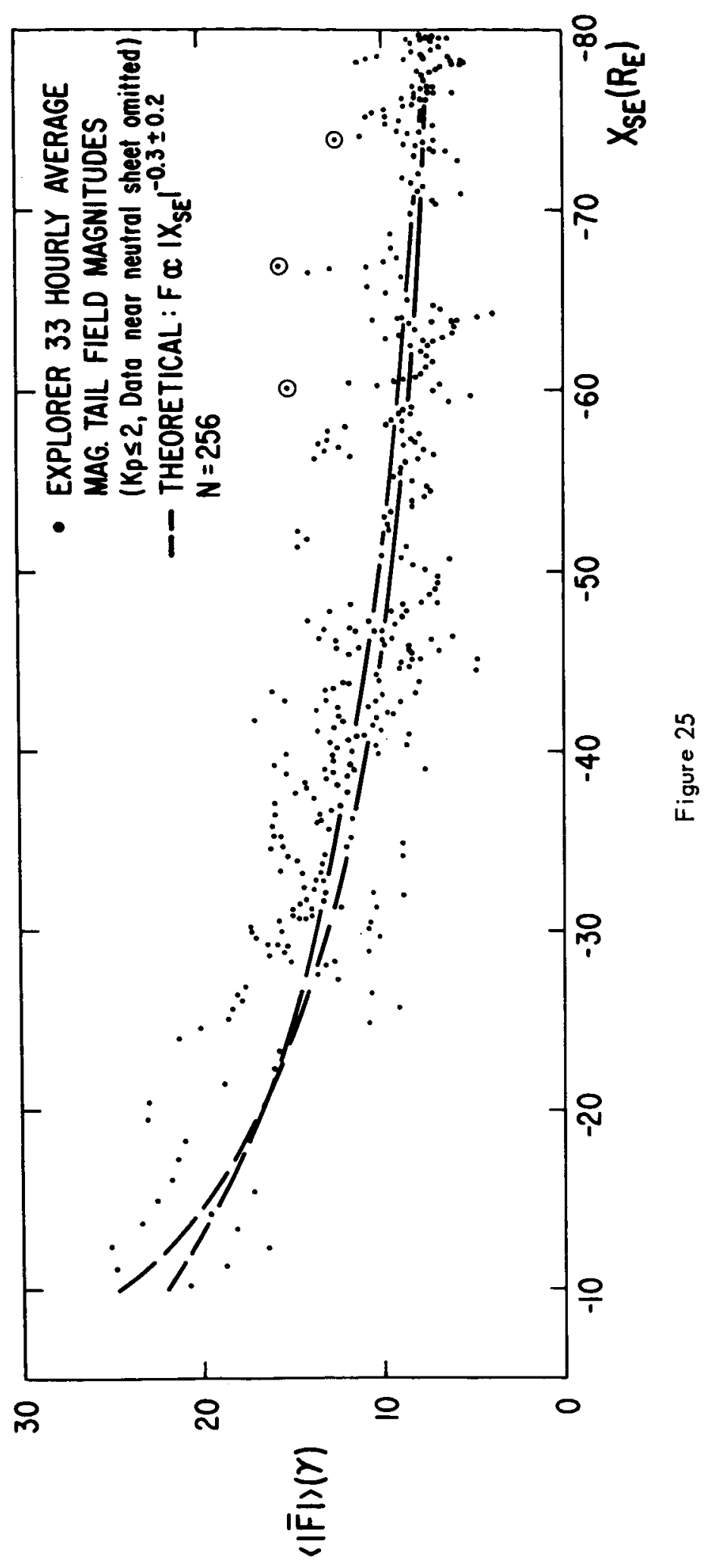

